\title{
SGLT2 Inhibitors: Cardiovascular Benefits Beyond HbA1c-Translating Evidence into Practice
}

\author{
Amar Ali · Steve Bain · Debbie Hicks · Phillip Newland Jones • \\ Dipesh C. Patel · Marc Evans · Kevin Fernando · June James • \\ Nicola Milne $\cdot$ Adie Viljoen · John Wilding (D) - As part of The Improving Diabetes Steering Committee
}

Received: May 9, 2019 / Published online: July 9, 2019

(C) The Author(s) 2019

\begin{abstract}
Cardiovascular disease (CVD), including heart failure (HF), is a leading cause of morbidity and mortality in people with type 2 diabetes mellitus (T2DM). CVD and T2DM share common risk factors for development and progression, and there is significant overlap between the conditions in terms of worsening outcomes. In assessing the cardiovascular $(\mathrm{CV})$ safety profiles
\end{abstract}

Enhanced Digital Features To view enhanced digital features for this article go to https://doi.org/10.6084/ m9.figshare.8276270.

A. Ali

Oakenhurst Medical Practice, Blackburn, UK

S. Bain

Diabetes Research Unit Cymru, Swansea University, Swansea, UK

D. Hicks

Medicus Health Partners, Enfield, UK

P. Newland Jones

University Hospitals Southampton NHS Foundation

Trust, Southampton, UK

D. C. Patel

Department of Diabetes and Endocrinology, Division of Medicine, University College London, London, UK

M. Evans

Department of Diabetes, University Hospital

Llandough, Llandough, UK of anti-diabetic drugs, sodium-glucose cotransporter-2 inhibitor (SGLT2i) therapies have emerged with robust evidence for reducing the risk of adverse CVD outcomes in people with T2DM who have either established CVD or are at risk of developing CVD. A previous consensus document from the Improving Diabetes Steering Committee has examined the potential role of SGLT2is in T2DM management and considered the risk-benefit profile of the class and the appropriate place for these medicines within the T2DM pathway. This paper builds on these findings and presents practical guidance for maximising the pleiotropic benefits of this class of medicines in people with T2DM in terms of

K. Fernando

North Berwick Health Centre, North Berwick, UK

J. James

University Hospitals of Leicester NHS Trust, Leicester, UK

N. Milne

CODES (Community Diabetes Education and Support), Manchester University NHS Foundation Trust, Manchester, UK
A. Viljoen
Department of Metabolic Medicine/Chemical
Pathology, Lister Hospital, Stevenage, UK
J. Wilding $(\bowtie)$
Obesity and Endocrinology Research, University of
Liverpool, Liverpool, UK
e-mail: J.P.H.Wilding@liverpool.ac.uk 
reducing adverse CVD outcomes. The Improving Diabetes Steering Committee aims to offer evidence-based practical guidance for the use of SGLT2i therapies in people with T2DM stratified by CVD risk. This is of particular importance currently because some treatment guidelines have not been updated to reflect recent evidence from cardiovascular outcomes trials (CVOTs) and real-world studies that complement the CVOTs. The Improving Diabetes Steering Committee seeks to support healthcare professionals (HCPs) in appropriate treatment selection for people with T2DM who are at risk of developing or have established CVD and examines the role of SGLT2i therapy for these people.

Funding: Napp Pharmaceuticals Limited.

Keywords: Anti-diabetic medicines; Cardiovascular disease (CVD); Clinical guidance; Heart failure (HF); Oral glucose-lowering medicines; Practical treatment selection; SGLT2 inhibitors (SGLT2i); Therapy choice; Type 2 diabetes mellitus (T2DM)

\section{THE ROLE OF THE IMPROVING DIABETES STEERING COMMITTEE}

The Improving Diabetes Steering Committee comprises a panel of clinical experts from across primary and specialist care, who meet with the objective of improving diabetes care. The Committee aims to ensure that HCPs who prescribe diabetes medicines have access to balanced and accurate information and evidence concerning type 2 diabetes mellitus (T2DM) medicines, with a specific focus on the sodium-glucose co-transporter-2 inhibitor (SGLT2i) class of treatments. The group is committed to providing healthcare colleagues with clarity concerning the evidence base supporting SGLT2i agents, highlighting the relative benefits and risks of these therapies. Educational materials and publications, such as the previous consensus document [1], provided by the panel are intended to increase confidence and understanding regarding the appropriate place of these medicines within the current UK
T2DM treatment paradigm. Due to the nature of the Committee, this article and related discussions are based on previously conducted studies and does not contain any studies with human participants or animals performed by any of the authors.

\section{TAKING TYPE 2 DIABETES OUT OF ISOLATION: THE IMPACT OF CARDIOVASCULAR DISEASE}

People with T2DM commonly live with multiple comorbidities, among which hypertension and cardiovascular disease (CVD) are the most common [2]. A systematic review of global clinical records from over 4.5 million people with T2DM (median duration 10 years) observed that CVD was a comorbidity in approximately one third of people with T2DM; of those, $29.1 \%$ had atherosclerosis, $21.2 \%$ had coronary heart disease (CHD), 14.9\% had heart failure (HF), 14.6\% had angina, 10.0\% had had a myocardial infarction (MI) and $7.6 \%$ had experienced stroke [3]. A recent cohort study, utilising data from the UK Clinical Practice Research Datalink, found a similar prevalence of these conditions in over 1.5 million people with T2DM in the UK [4]. In people with T2DM, elevated risk of peripheral arterial disease, ischaemic stroke, stable angina, HF and nonfatal MI was observed; there was no associated increased risk of arrhythmia or sudden cardiac death and an inverse relationship with abdominal aortic aneurysm and subarachnoid haemorrhage [4]. The fact that a large proportion of people with T2DM who are managed in routine practice have concomitant CVD raises the important question of whether it is effective to treat people by addressing each disease in isolation, or whether a holistic, personalised approach that takes shared risk factors into account can contribute to improved outcomes.

Overall, CVD is associated with significant morbidity and mortality in people living with T2DM, and may account for as many as half $(50.3 \%)$ of deaths in this population [3]. This is markedly higher than the global mortality rate for CVD of 31\% [5]. 
The burden of CVD in T2DM is considerable, both for people living with comorbid conditions and for the healthcare systems that support them. Globally, comorbid CVD contributes to $20-49 \%$ of the total cost of managing T2DM [6]. Compared with treating T2DM without CVD, median annual costs are 112\% higher with CVD, 107\% higher with coronary artery disease (CAD), 59\% higher with HF and 322\% higher with stroke [6].

Efficacious management of CVD risk and disease in people with T2DM is therefore a priority for both the individuals affected and for healthcare systems more broadly.

\section{THE RELATIONSHIP BETWEEN TYPE 2 DIABETES AND CARDIOVASCULAR DISEASE:
COMMON FACTORS}

T2DM and CVD share risk factors for the onset and worsening of disease [7]. The common pathophysiological processes include insulin resistance, chronic systemic inflammation, hypercoagulability, elevated blood pressure, dyslipidaemia and obesity [7-9]. Moreover, the presence of hyperglycaemia, which defines T2DM, is an independent risk factor for CVD [9]. Obesity is recognised as a risk factor for CVD and HF, and contributes to the development of insulin resistance [10-12]. In many cases, insulin resistance develops prior to onset of hyperglycaemia in T2DM, and both of these disorders increase the risk of developing CVD [13, 14]. Insulin resistance is therefore a key driver in the pathogenesis of CVD in people who present with HbA1c levels below the threshold for a diagnosis of type 2 diabetes. Increasing insulin resistance and consequent dysglycaemia should be recognised as risk factors for CVD; a study in nearly 12,000 patients without a history of diabetes demonstrated that asymptomatic hyperglycaemia was associated with higher risk of CV death compared with patients with low post-load glucose levels [15-17].

Insulin resistance is also involved in a complex interplay with multiple metabolic functions, and contributes to the pathophysiology of hypertension and dyslipidaemia [18]. Diabetic dyslipidaemia has been identified as an independent risk factor for $\mathrm{CHD}$ and stroke $[19,20]$.

These overlapping risk factors are common, which is reflected in the fact that a relatively high proportion $(>20 \%)$ of people with T2DM who are treated by HCPs in primary care have concomitant CVD [21].

\section{HEART FAILURE: A NEW FOCUS FOR CARDIOVASCULAR DISEASE IN DIABETES}

Previously, considerations around CV outcomes in people with T2DM have focused on the incidence of stroke, MI and CV death, which reflects the endpoints of the atherosclerotic disease process $[22,23]$. These three outcomes are commonly combined into a study endpoint called 3-point Major Adverse CV Events (3PMACE) [23]. Historically, HF is one of the most common and earliest presenting CV complications, and may develop independently of atherosclerosis [4, 22, 24]. Overall, 35-45\% of patients with chronic HF have T2DM [22], and diagnosis of $\mathrm{HF}$ is the first $\mathrm{CV}$ event in around $14 \%$ of people with T2DM [4]. Compared with the general population, the relative risk (RR) of developing HF in people with T2DM is twofold higher for men and fivefold higher for women [25]. Prior to the studies discussed below, HF was not included in primary outcome evaluations of cardiovascular outcomes trials (CVOTs) in T2DM $[26,27]$. The criteria for diagnosing HF have been inconsistent across clinical trials and baseline diagnosis of HF was not independently adjudicated in CVOTs; however, in the SGLT2i CVOT endpoint data presented here, HF was blindly adjudicated using criteria that are used in HF studies. The prevalence of people with T2DM with comorbid HF in primary care management is relatively common $(>22 \%$ of patients) [21]; it has also been recognised that HF may be underdiagnosed in routine care $[28,29]$.

Insulin resistance and hyperglycaemia are common factors in the development of both T2DM and HF, along with advancing age and 
inflammation [22, 30, 31]. Hyperglycaemia and insulin resistance are associated with multiple adverse metabolic effects; people with diabetes have elevated levels of free fatty acids that require high oxygen consumption to metabolise, potentially impacting myocardial performance, and leading to intracellular accumulation of toxic intermediate products [32]. Hyperglycaemia may also drive increased oxidative stress, damaging the myocardium, reducing antioxidant defence and contributing to endothelial dysfunction [32]. Morphological changes in the heart may be triggered by accumulation of advanced glycation end products in people with hyperglycaemia, leading to a loss of blood vessel elasticity; furthermore, hyperglycaemia may be associated with necrosis of myocytes, which leads to deposition of collagen and myocardial fibrosis [32]. In people without diabetes, HF risk increases if haemoglobin A1c (HbA1c) is above $5.5 \%$, and a $1 \%$ increase in $\mathrm{HbA1c}$ is associated with a $39 \%$ increase in HF. In $\mathrm{T} 2 \mathrm{DM}$, the risk of $\mathrm{HF}$ rises by $8-32 \%$ per $1 \%$ increase in HbA1c [33]; thus HF risk appears to be associated with HbA1c in those with and without diabetes, independent of other risk factors. Development of HF in people with T2DM is associated with significantly greater mortality than in those with T2DM who do not develop HF [34].

It has also been demonstrated that HF is a risk factor for the development of T2DM. Over 7.7 years of follow-up, T2DM developed across New York Heart Association (NYHA) severities I-III of HF: $17 \%$ of patients in class I, $15 \%$ in class II and $20 \%$ in class III ( $P$ for trend $=0.05$ ). There was a twofold increase in likelihood of having fasting blood glucose of at least $7 \mathrm{mmol} /$ L in patients with more severe HF (NYHA class III [17\%] versus class I [7.8\%] or II 8.7\%]) [35].

This bidirectional relationship between T2DM and HF in terms of disease risks and progression highlights the need for a selection of effective treatment strategies in routine practice that recognise both diseases. There is also an increasing burden of HF: in a study of 4 million patient records in the UK between 2002 and 2014, there was an estimated increase of $12 \%$ in individuals with newly diagnosed HF and a $23 \%$ estimated increase in the number of prevalent HF cases [36]. This trend highlights a need for a more effective strategy to prevent and manage HF in people with T2DM.

\section{THE ROLE OF GLYCAEMIC CONTROL IN MANAGING CARDIOVASCULAR RISK IN PEOPLE WITH TYPE 2 DIABETES}

Management of CV risk factors, including glucose, plays a key role in the treatment of people with T2DM. Meta-analyses of long-term studies have demonstrated the benefits of reducing HbA1c on CV outcomes [37, 38]. Retrospective analysis of the UK Prospective Diabetes Study (UKPDS) observed lower RR of stroke (12\%; $P=0.035)$, MI $(14 \% ; P<0.0001)$ and HF $(16 \%$; $P=0.021)$ per $1 \%$ reduction in HbA1c [38]. However, microvascular outcomes (37\% decrease per $1 \%$ HbA1c; $P<0.0001$ ) were improved to a greater extent with glycaemic control than macrovascular outcomes [38], and reducing HbA1c alone has less benefit in reducing risk or incidence of $\mathrm{MI}$, stroke and CVD than reducing blood pressure (BP) or improving lipid management [39].

Benefit may be obtained with a 'treat-to-target' approach for HbA1c in the management of $\mathrm{CHD}$, because a close correlation between the risk of CHD and increasing HbA1c has been observed. In a prospective study of 1626 people with T2DM and 1321 people without diabetes, the RR of CHD increased by 1.14 (95\% confidence interval [CI] 1.07-1.21) per percentage point increase in HbA1c, across all HbA1c values, and RR increased by 2.36 (95\% CI 1.43-3.90) per percentage point in patients without diabetes but with an HbA1c of more than $4.6 \%$ [40]. Similar increases in the RR of CHD per percentage point increase in HbA1c were seen in a large prospective trial of nearly 30,000 people with T2DM, across a range of baseline HbA1c values and independent of age, sex and smoking history [41]. For patients with renal disease, meta-analysis of tight glycaemic control has been associated with delayed onset of microalbuminuria (4 studies, 19,846 participants: RR $0.82, \quad 95 \%$ CI $0.71-0.93)$ and 
progression of microalbuminuria (5 studies, 13,266 participants: RR 0.59, 95\% CI 0.38-0.93), although there was less certain evidence for effect on progression to end-stage kidney disease [42].

However, there is a limit to the effectiveness of targeting glycaemic control alone, using traditional anti-diabetic medications for the management of CVD and HF [43, 44]. In addition, there are cautionary notes in the aggressive pursuit of lower HbA1c. No significant benefits were observed with intensive treatment of hyperglycaemia in the Action to Control Cardiovascular Risk in Diabetes (ACCORD; targeting an HbA1c below 6.0\%) study [45], the Action in Diabetes and Vascular Disease: Preterax and Diamicron MR Controlled Evaluation (ADVANCE; targeting an HbA1c below 6.5\%) study [46] or the Veterans Affairs Diabetes Trial (VADT; median HbA1c of 6.9\%) [47]. Notably, the ACCORD study reported a significant $22 \%$ increase [hazard ratio (HR) $1.22 ; 95 \%$ CI $1.01-1.46 ; P=0.04$ ] in total deaths in the intensively treated group compared with placebo [45]. Until the recent CVOT findings, metformin was the only diabetes therapy associated with a lower rate of all-cause mortality in people with T2DM and HF, compared with combinations with sulfonylurea or insulin therapy [48].

Given that T2DM is associated with independent risk factors for CVD, considerations beyond just elevated HbA1c need to be accounted for when developing management strategies [9]. Appropriate, early intervention is needed to improve CV outcomes in people with T2DM, with treatment goals beyond achieving glycaemic control alone.

When treating T2DM in a person with comorbidities, especially one at risk of CVD, HCPs need to consider a personalised approach that achieves glucose lowering and reduces $\mathrm{CV}$ risk factors [49]. Recent CV outcomes studies have demonstrated cardiovascular benefits in people receiving an SGLT2i therapy for the management of T2DM [50-56].

\section{WHY MIGHT SGLT2I TREATMENTS INFLUENCE CARDIOVASCULAR OUTCOMES?}

The mechanisms by which SGLT2is provide the benefits that have been observed in recent studies remain to be elucidated. Biomarkers and surrogate endpoints measured in these trials demonstrate that SGLT2 inhibition modifies many risk factors for CVD, beyond glucose levels, including BP, weight, visceral adiposity, hyperinsulinaemia, arterial stiffness, albuminuria, circulating uric acid levels and oxidative stress $[57,58]$. These factors are implicit in multiple pathways related to cardiorenal outcomes; SGLT2i treatments modulate natriuresis and glycosuria-excretion of sodium and glucose-which modify the factors in these pathways (Fig. 1) [58].

In the context of SGLT2 inhibition, there is considerable overlap between these factors; for example, SGLT2i-induced BP lowering may be associated with reduced arterial stiffness, natriuresis and weight loss. Reduction in weight and visceral fat deposition is most likely linked to glycosuria and consequent negative energy balance. Arterial stiffness has been linked to obesity, hypertension and hyperglycaemia. Thus, synergistic modulation of these factors via SGLT2 inhibition is proposed to play a significant role in the reduction of risk for the development of CVD [57, 58].

Several mechanisms of action have been proposed to explain the benefits of SGLT2i agents on the heart. To date, none of the proposed mechanisms have been able to unequivocally explain the CV benefit demonstrated in randomised clinical trials (RCTs). SGLT2 inhibition may improve cardiac metabolism and bioenergetics by elevating the production of ketones, which represent a more efficient myocardial energy source than fatty acids and which require less oxygen to metabolise, consequently improving myocardial oxygen efficiency. The myocardium is less flexible in its use of available energy sources when stressed by diabetes or HF, and this increased availability of ketones may allow for more efficient cardiac function [59]. In addition, the 


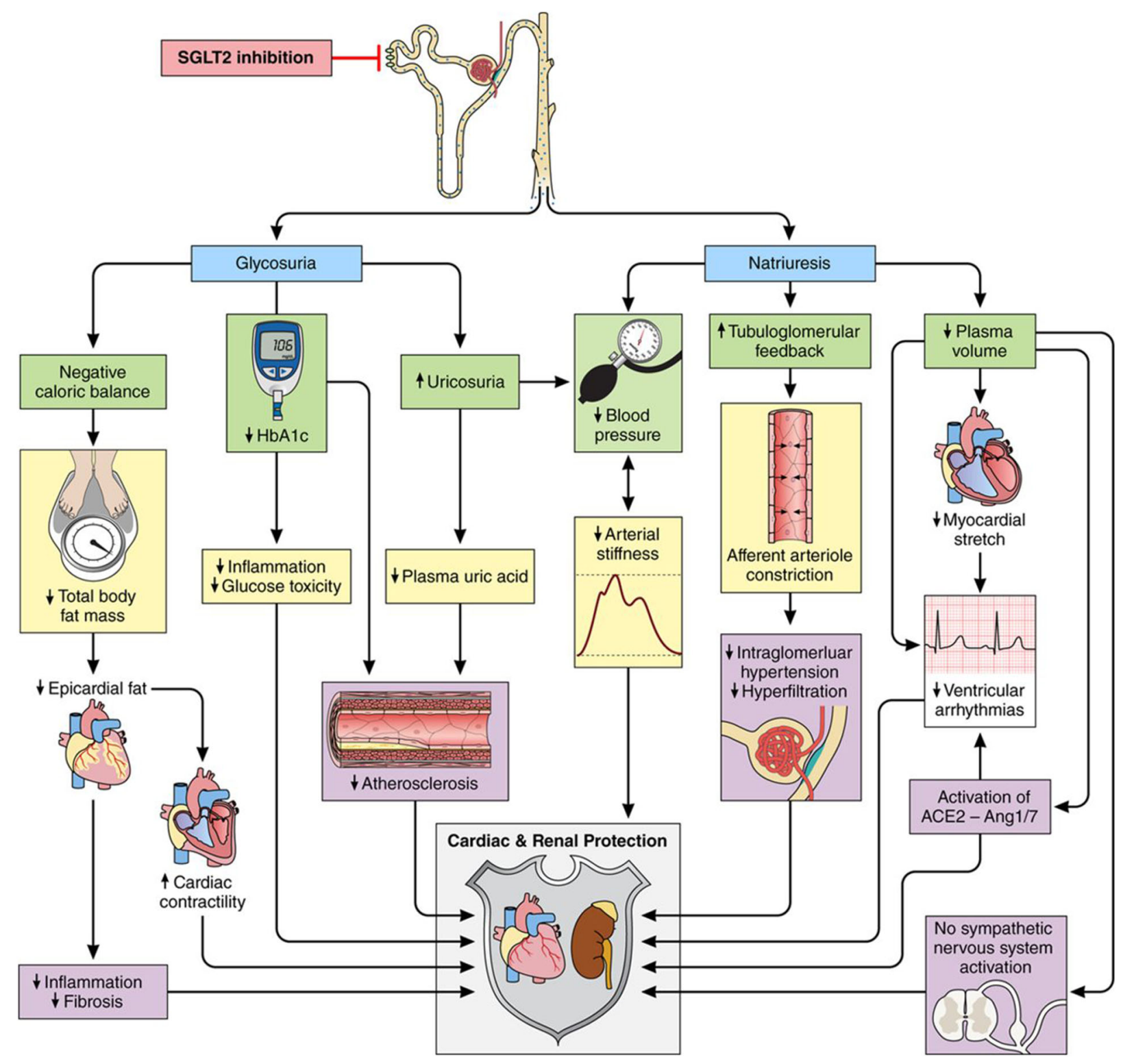

Fig. 1 Proposed pathways involved in cardioprotective role of SGLT2is. Multiple physiologic mechanisms related to cardiorenal outcomes are modulated with SGLT2i treatments. Adapted with permission from [58]

haemoconcentration that is typically induced by SGLT2 inhibition may result in improved tissue oxygen delivery, and a potential synergistic effect with the myocardial metabolic substrate shift [60]. More favourable ventricular loading conditions may also arise from reduced arterial stiffness and $\mathrm{BP}$, as a result of this osmotic diuresis and natriuresis [59]. Finally, SGLT2 inhibition may inhibit the $\mathrm{Na}^{+} / \mathrm{H}^{+}$ exchanger (NHE)-1 isoform in the myocardium, activation of which has been associated with elevated sodium and calcium levels in experimental models of HF [59]. Further investigation into the potential roles of modified cardiac fibrosis and cytokine production with SGLT2i treatment is underway [59].

It has been hypothesised that HF outcomes may be most sensitive to SGLT2i treatments. While atherosclerotic CVD (ASCVD) mechanisms may also be affected by SGLT2i treatment 
[59], the fact that a significant improvement in 3P-MACE was not achieved in the recent DECLARE-TIMI 58 study points to a tenuous link between ASCVD and SGLT2 inhibition in clinical outcomes [52]. However, the beneficial effect of SGLT2 inhibition on improving HF outcomes has been consistent [50-52].

SGLT2i treatments also appear to have positive renal benefits in reducing the rate of albuminuria progression and a reduced worsening of nephropathy. These benefits are postulated to arise from the restoration of tubuloglomerular feedback, which is a key mechanism in the kidney for the regulation of glomerular filtration rate (GFR) [61]. Other mechanisms, including amelioration of intrarenal hypoxia and subsequent anti-inflammatory effects have also been proposed.

Although SGLT2i treatments have a reduced glucose-lowering effect in people with reduced renal function, the renal benefits of SGLT2i agents appear to be preserved among people both with and without chronic kidney disease (CKD); these benefits include significant reduction in albuminuria and reduced incidence of worsening albuminuria $[62,63]$. The benefits on CVD outcomes with empagliflozin and canagliflozin have been demonstrated in patients with a range of baseline renal function capacities and across a range of urine albumin-creatinine ratios; the effects of dapagliflozin were similarly observed across a range of baseline eGFR values [5, 52, 64, 65]. Currently, SGLT2i treatment is indicated for initiation in people with T2DM and an eGFR $\geq 60 \mathrm{~mL} / \mathrm{min} / 1.73 \mathrm{~m}^{2}$ and should be discontinued if eGFR persistently falls below $45 \mathrm{~mL} / \mathrm{min} / 1.73 \mathrm{~m}^{2}$ [66-68]. The preserved efficacy of SGLT2 inhibition across a range of degrees of renal impairment may be of importance in treatment selection for people with T2DM, because CKD and T2DM are both associated with increased $\mathrm{CV}$ risk.

The efficacy of SGLT2i treatment will be further elucidated in the forthcoming Canagliflozin and Renal Endpoints in Diabetes with Established Nephropathy Clinical Evaluation (CREDENCE) trial (unpublished at time of paper development), which will prospectively assess the efficacy and safety of canagliflozin in clinically important renal and CV outcomes [69].
The primary composite endpoint is time to endstage kidney disease (ESKD), doubling of serum creatinine, and renal or CV death. At a planned interim analysis, the CREDENCE study demonstrated efficacy in achieving this primary endpoint and as a result the trial was stopped early [70]. Secondary endpoints include a CV composite endpoint of CV death, non-fatal MI, nonfatal stroke, hospitalisation for chronic HF and hospitalisation for unstable angina [69].

\section{WHAT EVIDENCE EXISTS FOR THE MANAGEMENT OF CVD RISK IN T2DM TREATMENT WITH SGLT2I AGENTS?}

For the past decade, there has been a regulatory requirement for new drugs intended for the treatment of T2DM to demonstrate that they 'do not increase CV risk to an unacceptable extent' and that phase III trials should include endpoints for non-fatal MI, stroke and CVD mortality [71]. This stipulation followed the suggestion that rosiglitazone was associated with a significant increase in the risk of MI and a borderline significant increase in $\mathrm{CV}$ death [72]. Following the requirement for anti-diabetic drugs to demonstrate that they do not increase $\mathrm{CV}$ risk, several cardiovascular outcome trials have been conducted and reported. Four trials with dipeptidyl peptidase-4 (DPP-4) inhibitors showed $\mathrm{CV}$ safety but no $\mathrm{CV}$ benefits [73-76]. Several meta-analyses suggested a possible increased risk of acute HF or hospitalised heart failure (HHF) with certain DPP-4 inhibitors versus placebo; however more research is required to explore these observations [77, 78]. In the glucagon-like peptide-1 receptor agonist (GLP-1 RA) class, four drugs have demonstrated significant improvements in $\mathrm{CV}$ safety outcomes versus placebo (liraglutide, semaglutide, albiglutide, dulaglutide) [79-82] and two have not (exenatide and lixisenatide) [83, 84]. To date, all three marketed SGLT2i medications have demonstrated benefits beyond glycaemic control, providing reductions in measures of CVD across populations with varying risk for, or degrees of, pre-existing CVD [50-52]. 
The Improving Diabetes Steering Committee has already commented on the risk-benefit considerations and the practical implications of the broad data from each pivotal SGLT2i trial available at the time of publication: EMPA-REG OUTCOME and Canagliflozin Cardiovascular Assessment Study (CANVAS); subsequently the Dapagliflozin Effect on CardiovascuLAR Events (DECLARE-TIMI 58) trial has provided additional data particularly for those at lower risk of CVD [1].

Here, the Committee considers the available evidence in the context of maximising benefit for people with T2DM and risk of CVD, with informed treatment selection and management strategy. A summary of study populations and selected endpoints is shown in Fig. 2. outcome versus placebo (HR 0.86; 95.02\% CI $0.74-0.99 ; P=0.04$ for superiority), a $38 \%$ RRR in CV deaths (HR 0.62; 95\% CI 0.49-0.77; $P<0.001$ ), a $35 \%$ RRR for HHF (HR $0.65 ; 95 \%$ CI $0.50-0.85 ; P=0.002$ ) and a $32 \%$ RRR in allcause mortality (HR 0.68 ; 95\% CI $0.57-0.82$, $P<0.001)$ [50]. The study observed no significant differences between empagliflozin and placebo in MI reports (4.8\% and 5.4\%, respectively) or stroke incidence $(3.5 \%$ and $3.0 \%$, respectively) [50].

These findings were expanded in a subanalysis of EMPA-REG OUTCOME, which examined outcomes in people with T2DM and confirmed CVD but differing HF status in those with and without HF at enrolment [85]. Empagliflozin reduced the rate of HHF in people

\begin{abstract}
At a glance: Summary of different patient populations in each pivotal SGLT2i CVOT.
The populations in EMPA-REG, the CANVAS programme and DECLARE-TIMI 58 differed in terms of what proportion of people had existing symptomatic CVD and what proportion did not have established CVD but were at risk of developing it [50-52].

- In EMPA-REG, all participants had established CVD.

- In the CANVAS trials, $65.6 \%$ of participants had established CVD and $34.4 \%$ had multiple risk factors.

- In DECLARE-TIMI 58, 40.6\% of participants had established CVD and 59.4\% had multiple risk factors.

These differences in populations may lead to different magnitudes of efficacy (a larger reduction in outcomes may be expected in a population with existing disease compared with a subpopulation without existing disease). DECLARE-TIMI 58 also had a relatively lower proportion of patients with more severe CVD, representing a population at lower risk of CVD and renal outcomes. However, insights into these populations can help inform the role of SGLT2i medications in primary and secondary prevention of CVD.
\end{abstract}

EMPA-REG OUTCOME examined the efficacy and safety of empagliflozin $10 \mathrm{mg}$ or $25 \mathrm{mg}$, compared with placebo, in addition to standard of care. The trial enrolled 7020 people with T2DM at high risk for CVD outcomes-all participants had established CVD [50]. EMPAREG OUTCOME reported a 14\% relative risk reduction (RRR) in the 3P-MACE primary stratified as low-to-average risk (5-year HF occurrence $<10 \%)$, high risk (10-20\%) and very high risk (>20\%), compared with placebo. Risk of CV mortality was similarly reduced in people with T2DM either with HF (HR 0.67 [0.47-0.97]) or without HF burden (HR 0.63 [0.48-0.84]) at baseline [85]. This study noted that in people with T2DM and established CVD, there was a 


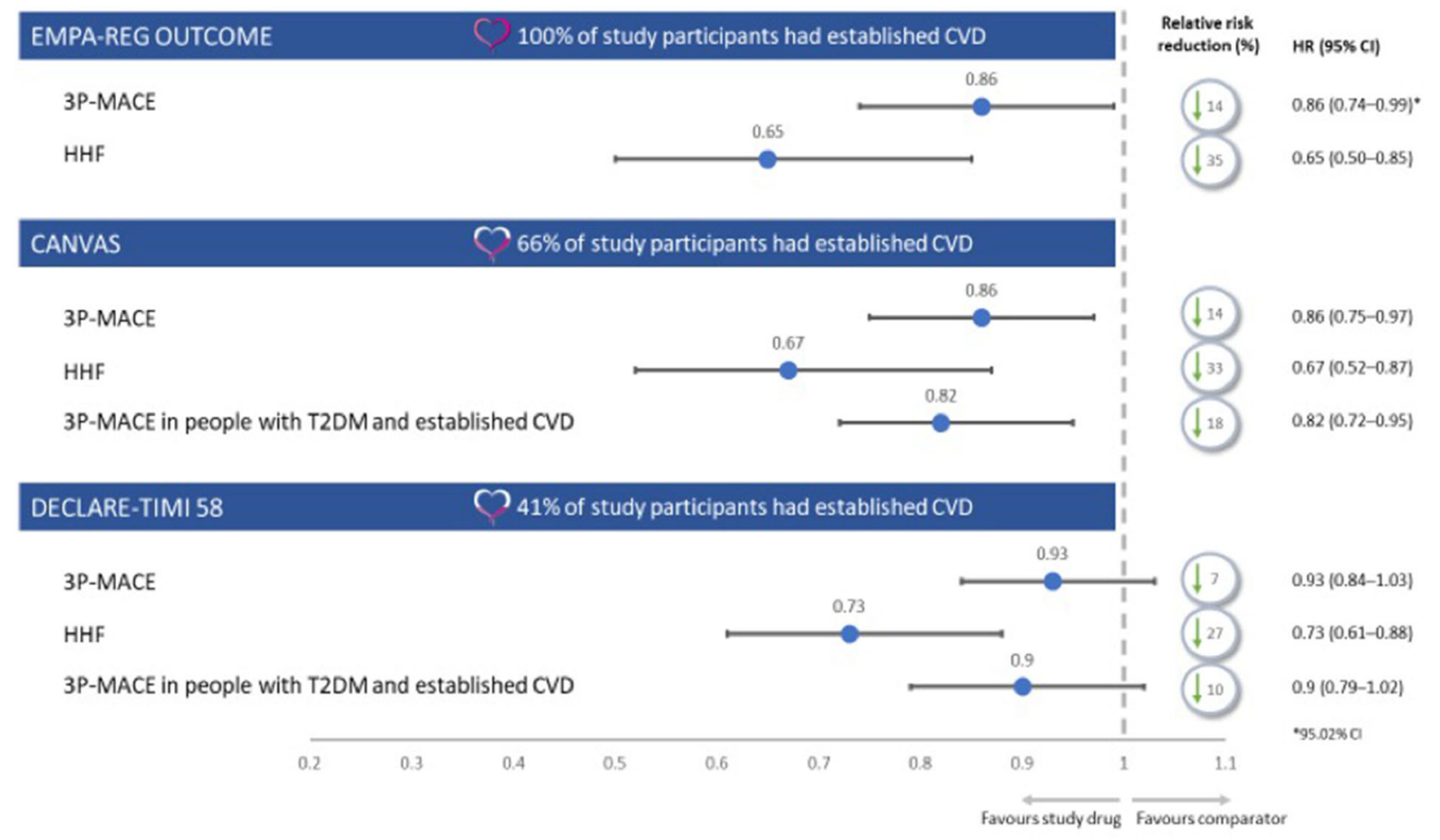

Fig. 2 Overview of trial population and selected cardiovascular endpoints in SGLT2i CVOTs [50-52]. This figure reports selected outcomes from three trials of an active comparator versus placebo in addition to standard of care, and is not a head-to-head trial outcome. 3P-MACE

high risk of mortality with baseline or incident HF (15.3\% in placebo) compared with people with no HF burden (4.2\% in placebo) [85]. Further analysis has observed that the primary endpoint benefits with empagliflozin were observed over a range of baseline HbA1c values and levels of renal function [86].

CANVAS examined the efficacy and safety of canagliflozin $100 \mathrm{mg}$ or $300 \mathrm{mg}$, compared with placebo, in addition to standard of care. The trial enrolled 10,142 people (across CANVAS and CANVAS-renal [CANVAS-R]) with T2DM, either with prior symptomatic $\mathrm{CV}$ events or at least two risk factors for CVD [51, 65]. CANVAS demonstrated a 14\% RRR in the 3P-MACE primary outcome versus placebo (HR 0.86; 95\% CI $0.75-0.97 ; P=0.02$ for superiority). A sub-analysis assessing HF reported that, compared with placebo, canagliflozin was associated with a significantly lower risk of composite $\mathrm{CV}$ death or HHF (HR 0.78; 95\% CI 0.67-0.91), as was fatal 3-point major adverse cardiovascular events, CI confidence interval, CVD cardiovascular disease, CVOT cardiovascular outcomes trials, HHF hospitalisation for heart failure, HR hazard ratio

or HHF (HR $0.70 ; 95 \%$ CI $0.55-0.89$ ) or HHF alone (HR 0.67; 95\% CI 0.52-0.87) [87].

Pre-specified sub-analysis of the primary endpoint by pre-existing disease observed an RRR of $18 \%$ in people with a history of CVD (HR 0.82 ; 95\% CI $0.72-0.95$ ), reflecting benefit in a more comparable patient population to EMPAREG [51].

DECLARE-TIMI 58 examined the efficacy and safety of dapagliflozin $10 \mathrm{mg}$, compared with placebo, in addition to standard of care. The trial enrolled 17,160 people with T2DM who either had previous CVD or no established CVD and with multiple risk factors for the development of CVD-this latter population accounted for 10,186 of the participants $[52,88]$. DECLARE-TIMI 58 had two co-primary efficacy analyses: 3P-MACE and composite HHF and $\mathrm{CV}$ death. Dapagliflozin did not reduce 3PMACE rate (8.8\% with dapagliflozin versus $9.4 \%$ with placebo; HR 0.93; 95\% CI 0.84-1.03; 
$P=0.17)$. Dapagliflozin did result in a lower rate of $\mathrm{CV}$ death or HHF versus placebo (4.9\% versus 5.8\%; HR 0.83; 95\% CI 0.73-0.95; $P=0.005$ ). This reduction in composite rate reflected a lower rate of HHF alone (HR 0.73; 95\% CI
$0.76 ; 95 \%$ CI 0.67-0.87) [52]. However, the Committee notes that care should be taken in extrapolating these data because formal statistical analysis of this endpoint ceased once the primary MACE endpoint was not met.

\section{Update on SGLT2i safety outcomes}

The Improving Diabetes Steering Committee has already commented on safety data from EMPA-REG OUTCOME and CANVAS [1].Since the publication of that document, DECLARE-TIMI 58 has released its safety data set.

Recent safety findings for dapagliflozin versus placebo included a higher rate of genital infections $(0.9 \%$ of study population versus $0.1 \%$ with placebo; $P<0.001)$, but no significant safety signal concerning fractures $(5.3 \%$ versus $5.1 \% ; P=0.59)$, amputation $(1.4 \%$ versus $1.3 \% ; P=0.53)$, or urinary tract infection $(1.5 \%$ versus $1.6 \%$; $P=0.54)$. Acute kidney injury was notably less frequent with dapagliflozin-treated people $(1.5 \%$ versus $2.0 \% ; P=0.002)$. Diabetic ketoacidosis was more common with dapagliflozin than with placebo $(0.3 \%$ versus $0.1 \%, P=0.02)$ [51]. Fournier's gangrene was rare, with one case in the dapagliflozin arm and five cases with placebo. Given the considerable differences in patient populations in EMPA-REG, CANVAS and DECLARE-TIMI 58, especially considering relative rates of previous disease and events $(0.6 \%$ of participants in DECLARE-TIMI 58 had previous amputations, compared with $2.3 \%$ in CANVAS) $[51,84]$, care will need to be taken in interpreting and generalising safety signals. These updates have been reflected in the prescribing tool previously developed by the Improving Diabetes Steering Committee, and updated in April $2019^{[1]}$.

0.61-0.88); there was no difference between dapagliflozin and placebo in rate of $\mathrm{CV}$ death (HR 0.98; 95\% CI 0.82-1.17) [52]. In a subgroup of patients with existing CVD, 3P-MACE was reduced to a greater extent than in the overall DECLARE-TIMI 58 population, although the improvement was still not statistically significant (HR 0.90; 95\% CI 0.79-1.02); this subgroup of patients is more comparable to the EMPAREG trial population [52]. In patients with prior MI, dapagliflozin was associated with a $16 \%$ RRR in 3P-MACE and a $2.6 \%$ absolute risk reduction versus placebo $(15.2 \%$ versus $17.8 \%$; HR $0.84 ; 95 \%$ CI $0.72-0.99 ; P=0.039$ ); there was no difference in MACE reduction in people without a prior MI [89].

Dapagliflozin was associated with improvement in the composite renal secondary endpoint $(\geq 40 \%$ eGFR reduction to $<60 \mathrm{~mL} / \mathrm{min}$ per $1.73 \mathrm{~m}^{2}$, end-stage renal disease, or renal or $\mathrm{CV}$ death) compared with placebo (events in $4.3 \%$ versus $5.6 \%$ of each arm, respectively; HR
These pivotal RCTs have demonstrated CVD or HF benefits with SGLT2i therapies in people with T2DM and at high risk of CVD. However, it is important to remember that these studies have highly selective populations to enable the study to be conducted under the carefully controlled conditions often necessary for statistical calculations, and many people who may be eligible for an SGLT2i in routine practice are excluded [90]. Real-world evidence (RWE) can complement RCT data and support HCPs in decision-making for the appropriate treatment of their patients.

\section{BROADENING THE EVIDENCE BASE: THE ROLE OF REAL-WORLD EVIDENCE IN INFORMING TREATMENT DECISIONS}

Real-world studies have the advantage of observing the safety and efficacy of drugs in 


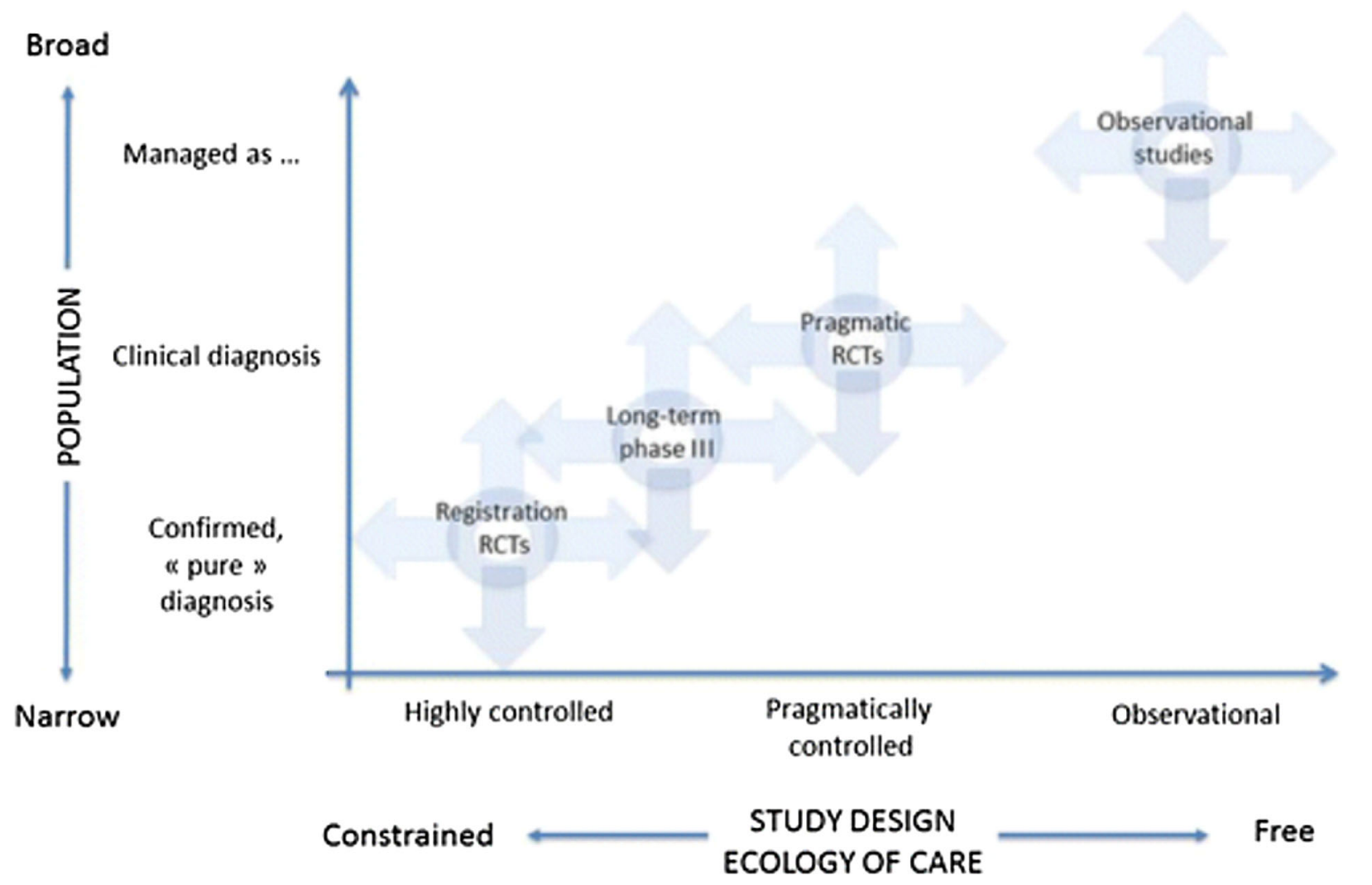

Fig. 3 Population characteristics of different types of therapeutic trial. Real-world observational evidence complements prospective data from randomised controlled trials by examining the safety and efficacy of drugs in a broader population that is representative of people most

broader populations that are more representative of the people most likely to be prescribed the studied drugs in daily clinical practice (Fig. 3) [91]. Real-world studies facilitate the confirmation of RCT findings against observations in routine clinical practice and can therefore help increase the external validity and generalisability of RCT data. RWE also has limitations: data capture is performed in an uncontrolled setting, which is less robust than in an RCT environment, and real-world studies may be more prone to, and less able to adjust for, confounding data than RCTs $[92,93]$.

Importantly, RWE can provide insight into how a drug performs in the real-world treatment of people and these findings, often in large study populations, can support HCPs in making appropriate, evidence-based, therapy choices. This is of particular relevance when likely to use the drug [91]. RCT randomised controlled trial. Reprinted with permission of the American Thoracic Society Copyright (C) 2019 American Thoracic Society. Roche et al. [91]. Annals of the American Thoracic Society is an official journal of the American Thoracic Society

considering benefits beyond glucose control, as the people observed in real-world studies will have typical comorbidities and concomitant treatments that may have resulted in them being excluded from RCTs [91].

\section{SGLT2I TREATMENTS IN ROUTINE PRACTICE: REAL-WORLD EVIDENCE FOR CARDIOVASCULAR BENEFITS IN PEOPLE WITH TYPE 2 DIABETES}

Three large recent real-world studies have observed the CV benefits of SGLT2i treatments in typical people with T2DM. In total, these studies provide RWE on outcomes in over one million people with T2DM. Furthermore, these studies examine outcomes similar to those reported in the RCTs described earlier. These 
studies also provide insight into class trends in outcomes with SGLT2is, which can support HCP decision-making. A summary of study populations and selected endpoints is shown in Fig. 4. for 374 days in the SGLT2i-treated cohort and 392 days in the oGLD cohort. CVD-REAL reported that SGLT2i treatment, versus oGLD in people with T2DM either with $(13 \%$ of the matched population) or without established

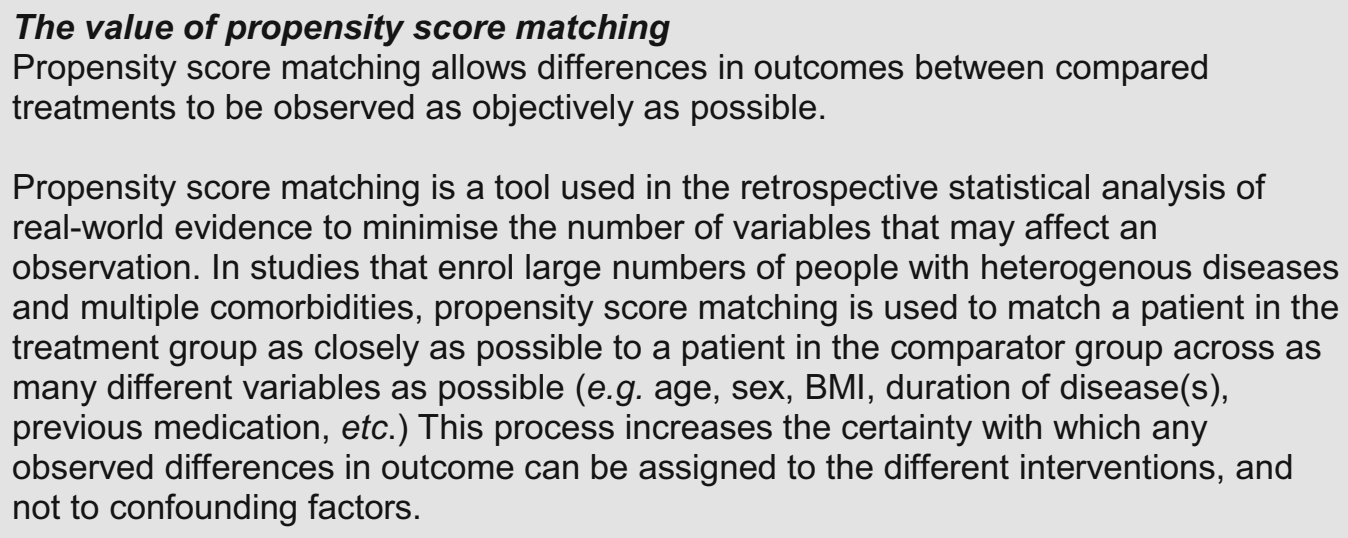

The Evidence for Cardiovascular Outcomes with Sodium Glucose Cotransporter-2 Inhibitors in the Real World (EASEL) study followed a propensity-matched population of 25,258 people with T2DM and established CVD for a mean duration of 1.6 years. EASEL assessed the benefits of SGLT2i compared with non-SGLT2i treatments. In the cohort prescribed an SGLT2i treatment, $58.1 \%$ received canagliflozin, $26.5 \%$ received empagliflozin and $15.4 \%$ received dapagliflozin [56]. For its primary composite endpoint (HHF and all-cause mortality), EASEL reported a lower event rate in SGLT2i-treated people than those treated with non-SGLT2i therapies [1.73 versus 3.01 events per 100 person-years (HR 0.57; 95\% CI 0.50-0.65)]. The SGLT2i-treated cohort, compared with the nonSGLT2i-treated cohort, also had better outcomes for all-cause mortality rate [1.29 versus 2.26 events per 100 person years (HR $0.57 ; 95 \%$ CI $0.49-0.66 ; P<0.0001)]$, HHF $[0.51$ versus 0.90 events per 100 person years (HR $0.57,95 \%$ CI 0.45-0.73; $P<0.0001)]$ and MACE [2.31 versus 3.45 events per 100 person years (HR 0.67; 95\% CI 0.60-0.75)] [56].

The CVD-REAL study followed a propensitymatched population of 309,056 people with T2DM, newly initiated on either an SGLT2i agent or 'other glucose-lowering drugs' (oGLD)
CVD, was associated with lower risk of HF (HR $0.72 ; 95 \%$ CI $0.62-0.83$ and HR 0.61; 95\% CI $0.48-0.78$, respectively) and composite $\mathrm{HF}$ and death (HR 0.63; 95\% CI 0.57-0.07 and HR 0.56; 95\% CI $0.50-0.62$, respectively). There was a reduction in overall death in people with T2DM, with or without pre-existing CVD (HR 0.56 ; $95 \%$ CI $0.44-0.70$ and HR 0.56 ; $95 \%$ CI 0.50-0.63, respectively) [53]. In CVD-REAL 2, $27 \%$ of participants had established CVD [54]. Compared with oGLD therapy, SGLT2i treatment was associated with lower rates of HHF (HR 0.64; 95\% CI 0.50-0.82; $P<0.001$ ), death (HR 0.51; 95\% CI 0.37-0.70; $P<0.001$ ), MI (HR $0.81 ; 95 \%$ CI $0.74-0.88 ; P<0.001)$ and stroke (HR 0.68; 95\% CI 0.55-0.84; $P<0.001$ ) $[53,54]$. CVD-REAL Nordic assessed the benefits of dapagliflozin compared with DPP-4 inhibitors in 40,908 propensity-matched people (23\% with established CVD), newly initiated on treatment, with a follow-up of 0.95 years. Dapagliflozin was associated with a lower risk of MACE (HR 0.79; 95\% CI 0.67-0.94) and HHF (HR 0.62; 95\% CI 0.50-0.77) compared with the DPP-4 inhibitor group. Compared with DPP-4 inhibitor therapy, dapagliflozin was associated with non-significantly lower risk of non-fatal MI, non-fatal stroke and CV mortality [94]. 
OBSERVE 4-D examined outcomes in 142,800 new users of canagliflozin, 110,897 new users of other SGLT2i agents and 460,885 new users of non-SGLT2 $i$ therapies in a retrospective cohort analysis using observational databases, with a relatively short follow-up of 60-100 days. Of the subgroup of participants with established CVD, 43,043 were new users of canagliflozin, 31,011 were new users of other SGLT2i and 141,579 were new users of non-SGLT2i therapies [55]. OBSERVE 4-D reported that canagliflozin was associated with a reduction in HHF risk compared with non-SGLT2i medicines (HR $0.39 ; 95 \%$ CI $0.26-0.60 ; P=0.01$ ); there was no difference in risk reduction between canagliflozin and other SGLT2i agents (on-treatment HR 0.70; 95\% CI 0.30-1.63) [55]. assess and support the management of people with T2DM, considering future CVD outcomes.

\section{PRIMARY AND SECONDARY PREVENTION: WHEN MIGHT INITIATION OF AN SGLT2I THERAPY PROVIDE BENEFIT?}

Several trials have now released data for people both with established pre-existing disease and for people with no previous established symptomatic CVD, but with multiple risk factors. The benefits of SGLT2i treatment are robustly supported in secondary prevention of CVD and HF, with an increasing wealth of evidence demonstrating significant benefits in people with no

At a glance: Summary of different patient populations in the RWE studies described. As with the RCTs for SGTL2i outcomes, the RWE studies also had different proportions of participants with established CVD included in their analyses, after propensity score or cohort matching [53-56, 86].

- In EASEL, all participants had established CVD [56].

- In CVD-REAL, 13\% of matched participants had established CVD [53].

- In CVD-REAL 2, 27\% of matched participants had established CVD [54].

- In CVD-REAL Nordic, 23\% of matched participants had established CVD [94].

- In OBSERVE 4-D, 30\% of matched participants had established CVD [55].

A recent analysis noted that while the absolute values and magnitude of treatment effects will differ to some degree between RCT data and RWE for SGLT2i therapies-reflecting the different patient populations-the trends in CVD outcomes are similar and consistent [95].

Initial insights from the EMPRISE study, assessing the real-world efficacy in reducing HHF in people with T2DM (with or without existing CVD) with empagliflozin against DPP-4 inhibitors, have shown a $44 \%$ reduction in favour of the SGLT2i treatment [96]. As data emerge from routine practice, particularly from studies comparing active treatment arms, HCPs will have a greater evidence base from which to established CVD or HF [50-54, 56, 59, 85, 97].

A recent meta-analysis across the three published SGLT2i CVOT trials (EMPA-REG, CANVAS and DECLARE-TIMI 58) provided further support for the benefits of SGLT2i treatment in patients with existing CVD; however, it observed no role in reducing MACE in those without pre-existing CVD, and noted more emphasis on HF outcomes [97]. In terms of reduction of MACE, there was an overall risk reduction of $14 \%$ (HR 0.86 ; 95\% CI $0.80-0.93$ ) in patients with established CVD, but no improvement (HR 1.00; 95\% CI 0.87-1.16) in patients with risk factors only. Reduction in composite HHF and CVD favoured SGLT2i 


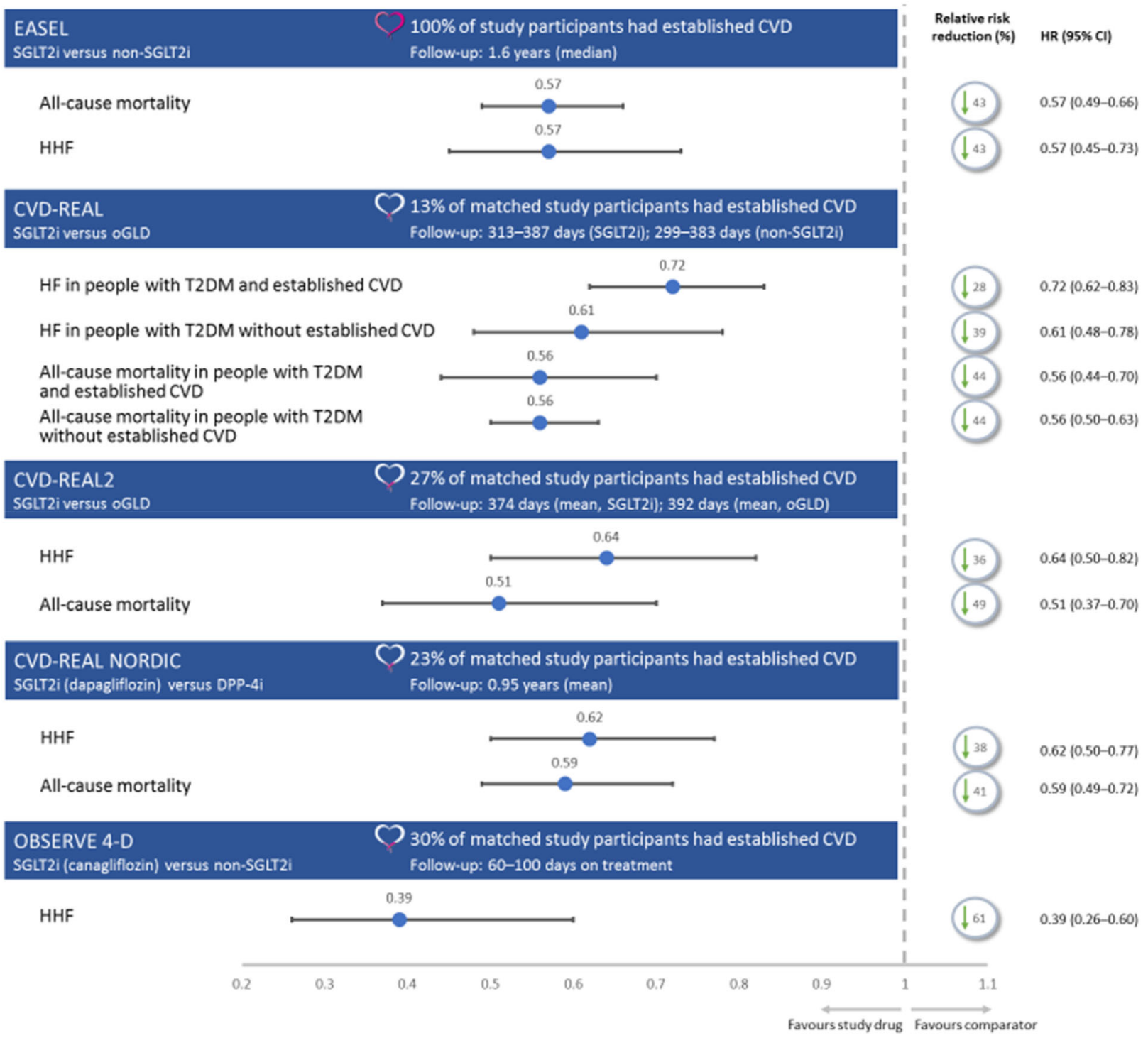

Fig. 4 Overview of trial population and selected cardiovascular endpoints in real-world evidence studies [53-56, 94]. This figure reports selected outcomes from four trials and is not a head-to-head trial outcome. CI

treatment over placebo in people with established CVD (HR 0.76; 95\% CI 0.69-0.84) and without established disease but multiple risk factors (HR 0.84; 95\% CI 0.69-1.01; $p$ for interaction 0.41; Fig. 5). The Committee notes that the findings for this composite endpoint were most likely driven by a reduction in HHF. Reduction of MACE appeared to be independent of renal function. However, benefits in HF confidence interval, CVD cardiovascular disease, DPP-4i dipeptidyl peptidase- 4 inhibitor, HF heart failure, HHF hospitalisation for heart failure, HR hazard ratio, oGLD other glucose-lowering drug

were greater in people with relatively reduced renal function [97].

It is the opinion of the Improving Diabetes Steering Committee that for people with T2DM and risk factors for CVD, regardless of the presence of established CVD or HF, that the early initiation of an SGLT2i treatment provides people with T2DM with an optimum management strategy by reducing hyperglycaemia, aiding weight loss and lowering systolic BP. 
a Meta-analysis of MACE outcomes stratified by presence of established CVD

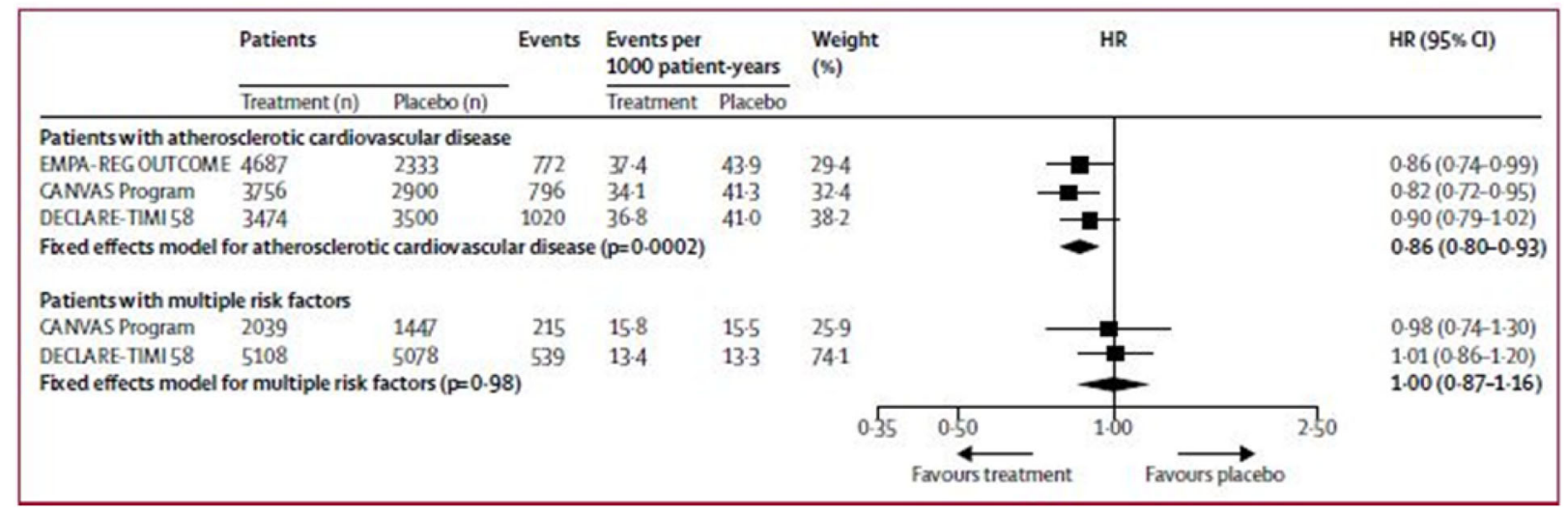

\section{b Meta-analysis of HHF and CV death stratified by presence of established CVD}

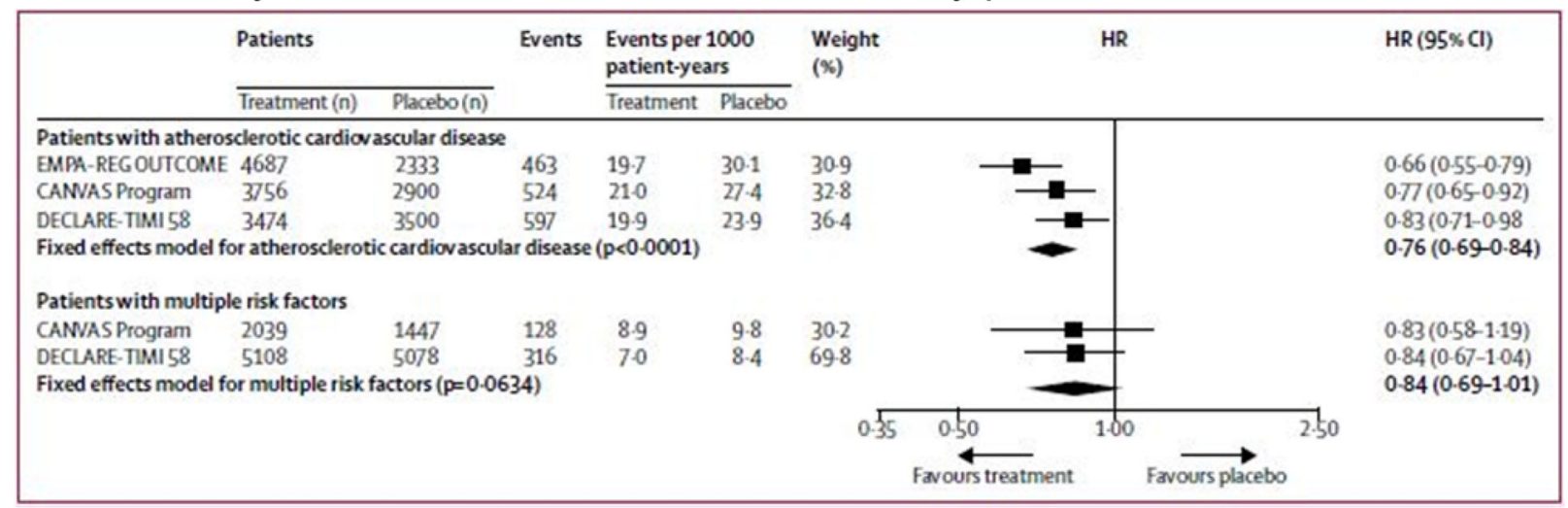

\section{c Meta-analysis of HHF and CV death stratified by history of HF}

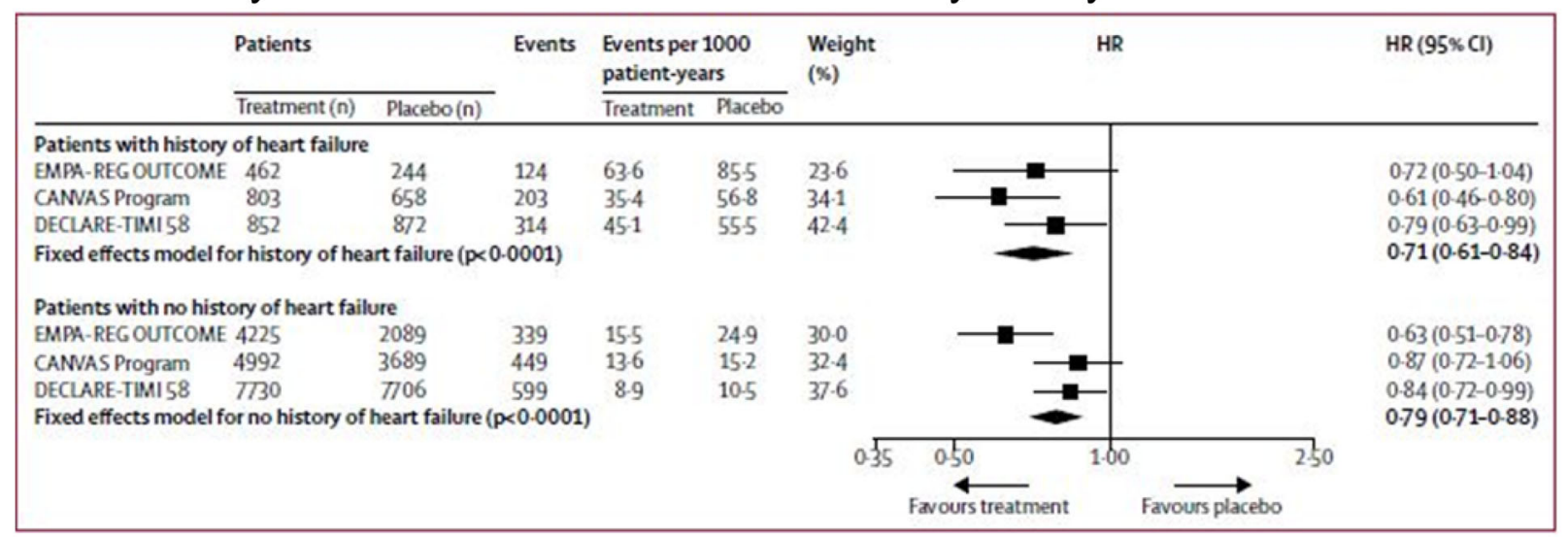

Fig. 5 SGLT2i treatment efficacy in people both with and without established CVD or HF [97]. a Meta-analysis of MACE outcomes stratified by presence of established CVD. $\mathbf{b}$ Meta-analysis of HHF and CV death stratified by presence of established CVD. c Meta-analysis of HHF and $\mathrm{CV}$ death stratified by history of HF. Adapted with permission from Zelniker et al. [97] 


\section{HOW IS THIS EVOLVING EVIDENCE- BASE BEING REFLECTED IN PRACTICAL GUIDELINES?}

A role for SGLT2i treatments in the management of T2DM is acknowledged in the current National Institute for Health and Care Excellence (NICE) clinical guidelines on T2DM management [98]. However, triple therapy option in these guidelines is limited to use with metformin and a sulfonylurea. Current NICE guidelines do not yet offer advice on selection of T2DM treatment according to CVD risk [99]. Recent Scottish Intercollegiate Guidelines Network (SIGN) guidelines recommend considering the evidence base for initiation of an SGLT2i or GLP-1 receptor agonist for patients with existing CVD, but do not offer advice on the specific selection of these drugs [100].

The American Diabetes Association (ADA)/ European Association for the Study of Diabetes (EASD) panel has issued a consensus statement on the use of anti-diabetic medicines when considering outcomes beyond glycaemic control alone (ASCVD, HF, CKD, weight management and control of hypoglycaemia; Fig. 6) [101]. This consensus represents a welcome advance in personalising T2DM management. However, the Improving Diabetes Steering Committee recognises that the ADA/EASD position statement has limitations in that it may be difficult for clinicians to decide which disease category has priority. For example, in a patient with both HF and ASCVD, it is difficult to assess whether an SGLT2i or a GLP-1 RA should be prescribed, especially with an absence of supportive evidence, in which case options should be discussed with people with T2DM and a decision made on an individual basis. The Committee suggests that in the period before NICE guidelines are updated to reflect the CVD and HF considerations of treatment selection, wider implementation of the ADA/EASD consensus would be of value in improving outcomes.

Local guidelines may not always reflect emerging data; moreover, they need considerable resource allocation to be updated, and updates are even more challenging in rapidly evolving treatment areas. It would therefore be valuable to have broader and more accessible advice for the use of SGLT2i treatments in people with T2DM at risk of CVD. This Improving Diabetes Steering Committee consensus paper aims to offer practical advice to HCPs in the selection of treatment as an add-on to metformin and seeks to facilitate personalisation of medicine for people with T2DM and at risk of developing CVD.

\section{OVERCOMING TREATMENT COMFORT IN TYPE 2 DIABETES MANAGEMENT: AND PREPARING FOR NEW TREATMENT APPROACHES}

It is well understood that there is significant 'inertia' in the management of people with T2DM, with treatment escalation often taking many years-leaving people with suboptimal glucose control [102]. As HCPs look beyond a glucocentric management strategy for people with T2DM at risk of CVD there are several challenges to adopting new treatment approaches, including a lack of familiarity with newer agents, limited prescribing options through local formulary practice and a lack of flexibility within prevailing local guidelines. Expanding the physician knowledge base and increasing awareness in routine practice of the performance of newer therapeutic options is key in overcoming these barriers. Demonstrating the efficacy of SGLT2i therapies in CVD outcomes in the context of other common CVD drugs may help physicians appreciate the magnitude of CVD benefit offered by this class.

A recent study noted that the number needed to treat (NNT) to see a benefit in all-cause death compared favourably for empagliflozin against simvastatin or ramipril [103]. When published data for CV death, HHF and all-cause mortality were examined in a non-head-to-head illustrative comparison, SGLT2i therapies have favourable $\mathrm{HF}$ reductions compared with angiotensin-converting enzyme inhibitors (ACEi) and angiotensin receptor blockers (ARBs), with each drug compared to placebo 


\section{CHOOSING GLUCOSE-LOWERING MEDICATION IN THOSE WITH ESTABLISHED ATHEROSCLEROTIC CARDIOVASCULAR DISEASE (ASCVD) OR CHRONIC KIDNEY DISEASE (CKD)}

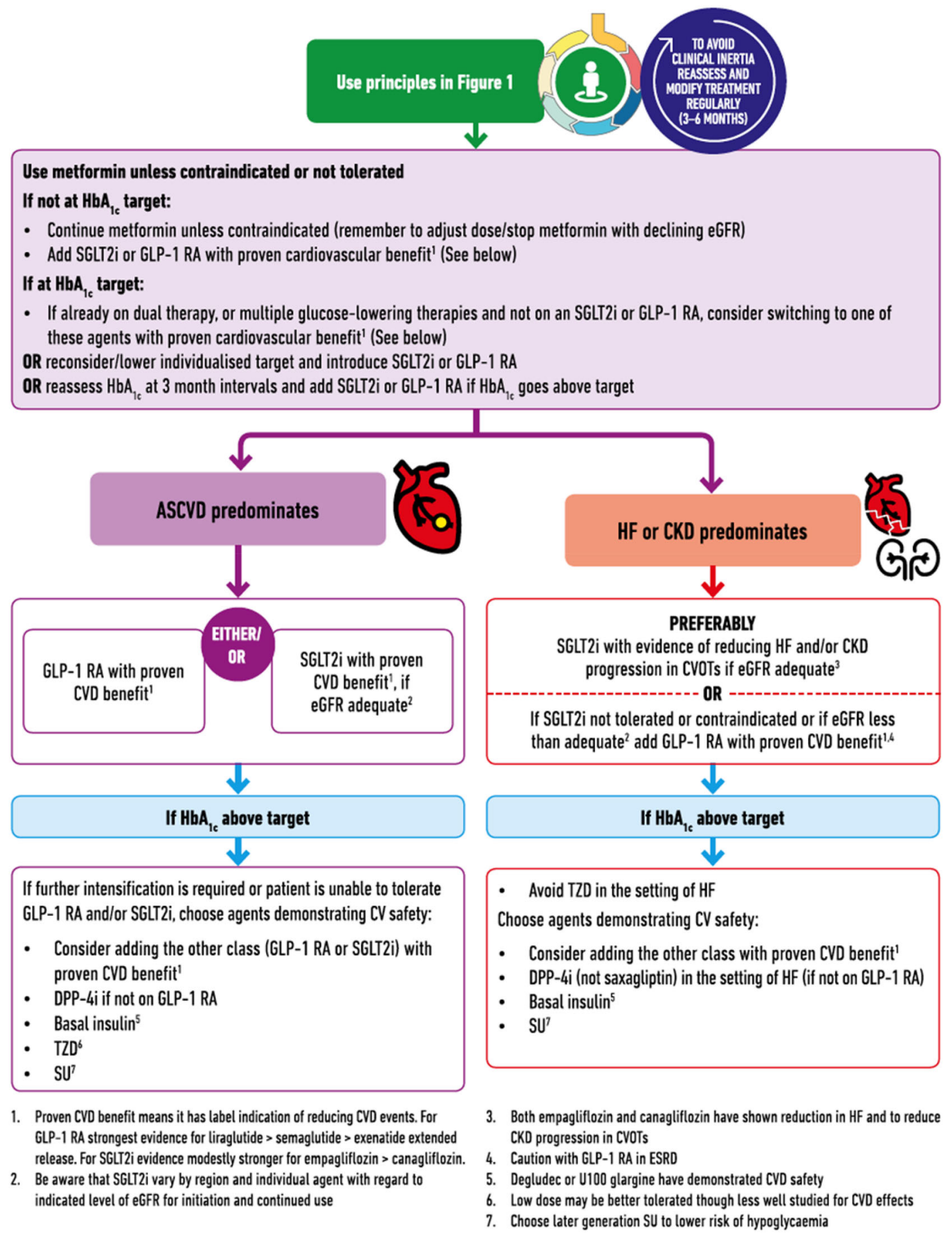

Fig. 6 ADA/EASD consensus on treatment selection in T2DM when considering CVD or renal outcomes. Adapted with permission from [101] 


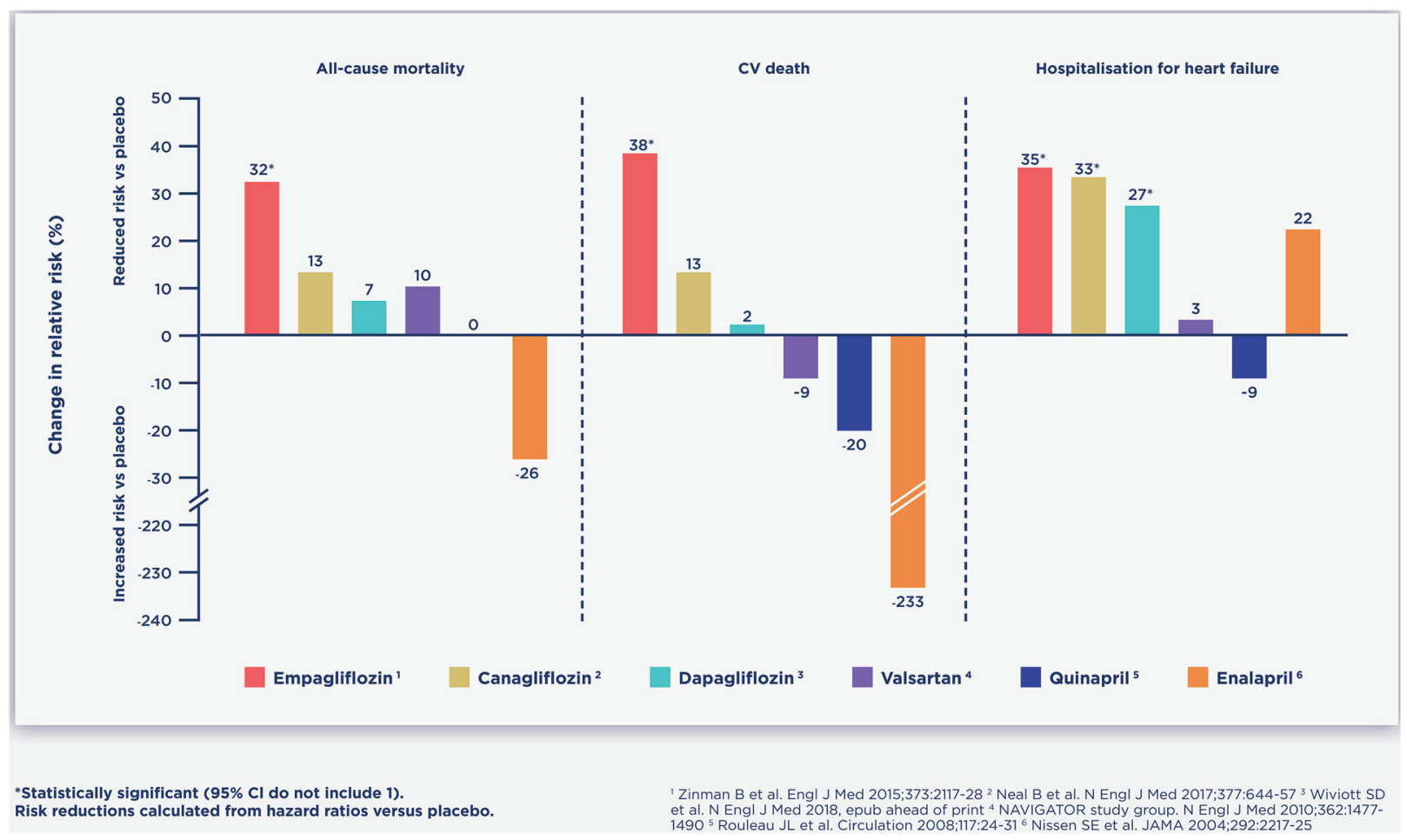

Fig. 7 Illustrative efficacy of SGLT2i treatments in the reduction of CVD risks in the context of commonly used CVD drugs

(Fig. 7). Given the comfort with which HCPs consider the prescription of these routine drugs, the Committee notes that early and appropriate intervention with SGLT2i therapies may yield economical and clinically meaningful benefits in people with T2DM at risk of CVD.

\section{PRACTICAL ADVICE: THE USE OF SGLT2I TREATMENT IN PEOPLE RECEIVING DIURETICS}

There is opinion that SGLT2i agents are broadly appropriate to use alongside diuretics, with routine monitoring of renal function, especially in patients receiving loop diuretics [104]. Current prescribing advice notes that empagliflozin may increase the diuretic effect of thiazide and loop diuretics and may increase the risk of dehydration and hypotension [67], and canagliflozin and dapagliflozin are not recommended for use in patients receiving loop diuretics $[66,68]$.
An analysis of the EMPA-REG OUTCOME trial suggests that the significant benefits of empagliflozin on HHF were seen in people not receiving diuretics (including loop diuretics) at baseline, but not in those that were [105]. This suggests that the diuretic action of SGLT2i agents may play a key role in the improvements these agents provide on HF outcomes [105]. A sensitivity analysis noted that empagliflozin had a positive effect on 3P-MACE in patients either using loop diuretics (HR 0.91; 95\% CI 0.67-1.23) or not (HR 0.84; 95\% CI 0.71-1.00) at baseline, and similar significant reduction in $\mathrm{CV}$ death in those receiving loop diuretics (HR 0.60 ; 95\% CI 0.40-0.90) or not (HR 0.63; 95\% CI 0.48-0.82) at baseline [85].

Although the primary pharmacological action of SGLT2i agents occurs in the proximal convoluted tubule (the location of the SGLT2 transporter), it has been suggested that the diuretic action that they induce could be a secondary downstream effect of altered sodium balance in the loop of Henle, meaning that 
SGLT2i treatments could be considered to act similarly to loop diuretics [105]. A study in healthy volunteers has demonstrated a mutual adaptive synergy between a loop diuretic (bumetanide) and dapagliflozin, suggesting that SGLT2i medications may be helpful in HF patients with resistance to loop diuretics [106]. A further, similar study examining the effects of SGLT2 inhibition in combination with the loop diuretic furosemide on diuresis, with the hypothesis that SGLT2 inhibition may augment the effects of loop diuretics, is ongoing [107].

If loop diuretic drugs (including SGLT2i medications) are administered alone, sodium reabsorption in the distal tubules of the nephrons is enhanced in a compensatory manner, limiting diuresis [105]. However, concomitant administration of loop diuretics with thiazide diuretics, which act on the distal tubules, may result in massive diuresis, hypotension and/or dehydration [105]. For this reason, HCPs initiating an SGLT2i therapy in a person receiving a combination antihypertensive therapy that includes a diuretic may choose to reduce or discontinue loop or thiazide diuretic before commencing the SGLT2i treatment; this consideration is of particular importance in the elderly.

The Improving Diabetes Steering Committee reiterates its previous practical advice in relation to co-prescription of SGLT2i with diuretics [1]:

- Check electrolyte and renal function for all individuals prescribed SGLT2i therapy. This is particularly important for people on diuretic therapies.

- Conduct routine electrolyte and renal function measurements, as appropriate for individual circumstances, comorbidities and concomitant medications, checking 1 month after initiation of an SGLT2i in people receiving diuretics. A modest reduction in eGFR of $3-5 \mathrm{~mL} / \mathrm{min} / 1.73 \mathrm{~m}^{2}$ may be expected following the initiation of SGLT2i therapy [108], as is the case for other medications such as ACEis.

- Consider reducing or stopping diuretic medicines for treatment of oedema or hypertension, especially if blood pressure is well controlled.
- Review medicines regularly and de-escalate therapy where possible, in line with NHS Scotland 2018 polypharmacy guidance.

\section{MAXIMISING BENEFITS IN THE TREATMENT OF TYPE 2 DIABETES: BEYOND HBA1C}

Treatment with SGLT2i medication has demonstrated efficacy in attaining glycaemic targets, for which the class is currently indicated [66-68]. The SGLT2i class is associated with weight loss and lowering of BP, no increase in hypoglycaemia and, via a number of mechanisms, has increasingly robust evidence for the reduction of CVD, HF hospitalisation and CV death, in people with and without pre-existing CVD [50-56, 85, 97]. Although the evidence on effects on overall mortality and ASCVD are still evolving, there is a demonstrated class effect showing benefit in HF. Treatment of T2DM with SGLT2 inhibition has been associated with significant reduction in albuminuria and reduced incidence of worsening albuminuria [62, 63].

SGLT2i treatments are well tolerated and have a favourable risk-benefit profile for many people with T2DM [1]. In practice, SGLT2i therapies appear to have a meaningful impact, with an NNT for several CVD outcomes similar to those of commonly prescribed drugs for the management and prevention of CVD [103].

As HCPs seek to personalise medicine for people with T2DM, it is appropriate to not only take a glucocentric approach. An approach of 'treating the person, rather than a disease' is of particular importance in managing T2DM, where multiple comorbidities are common. Treating each condition in isolation may lead to missed opportunities in improving overall outcomes for people with T2DM.

The Improving Diabetes Steering Committee suggests that SGLT2i therapies, for many people with T2DM and at risk of CVD, may represent a logical first escalation from metformin.

While glucose control remains the cornerstone of diabetes management, including selecting appropriate HbA1c targets and aggressiveness of therapy in consultation with 
the patient, there are clearly outcome benefits beyond attainment of glycaemic targets. It is prudent therefore to consider the CVD risk factors people with T2DM may have and then consider treatment options that take these factors into account. This is important considering the shared risk factors, particularly elevated blood pressure, dyslipidaemia and obesity, for developing CVD and T2DM.

\section{SUMMARY AND PRACTICAL CONSIDERATIONS}

Recent evidence from CVOTs and real-world studies provides robust support for the initiation of SGLT2i medication in people with T2DM and who either have established CVD or are at risk of developing CVD, and HF in particular, or at risk of renal decline and progression into CKD. It has been demonstrated that SGLT2i treatment in T2DM is efficacious in reducing HbA1c and is well tolerated. In addition, SGLT2i treatment has secondary benefits in terms of weight loss and lowering BP and has minimal risk of increased incidence of hypoglycaemia.

In selecting efficacious treatment for people with T2DM and risk of CVD, the ADA/EASD consensus statement notes that both SGLT2i and GLP-1 RA interventions may be appropriate, and recommend determining the existence of established CVD, or the probable risk of developing CVD or CKD. In people with T2DM and established ASCVD, either a GLP-1 RA or SGLT2 inhibitor, with demonstrated efficacy in improving CV outcomes, can be selected, post metformin. Where the risk of developing CKD or HF is the HCP's main concern, the initiation of SGLT2i therapy is recommended. If SGLT2i treatment is not tolerated or not appropriate (the patient has an eGFR outside of the licensed range, for example), then a GLP-1 RA with demonstrated CV outcome benefit should be used [101].

Here, the Improving Diabetes Steering Committee provides practical advice on the use of SGLT2i treatment in four major groups of people with T2DM: people with established CVD, people at high risk for developing CVD, people at lower risk of developing CVD and frail people. Level of evidence was graded according to ADA standards (Table 1). A simple 'traffic light' approach to clinical considerations in these populations is provided in Table 2.

In people with T2DM and established CVD, SGLT2i treatment provides clear benefits in reduction of adverse CVD outcomes. Unless contraindicated, the Committee advises that SGLT2i treatment is recommended as an oral treatment of choice, in combination with lifestyle and other glucose-lowering drugs as

Table 1 ADA evidence-grading system for “Standards of Medical Care in Diabetes" [109]

Level of Description

evidence

A Clear evidence from well-conducted, generalisable RCTs that are adequately powered, including:

- Evidence from a well-conducted multicentre trial or meta-analysis that incorporated quality ratings in the analysis

- Compelling non-experimental evidence

B Supportive evidence from well-conducted cohort studies

Supportive evidence from a well-conducted case-control study

C Supportive evidence from poorly controlled or uncontrolled studies

Conflicting evidence with the weight of evidence supporting the recommendation

E Expert consensus or clinical experience

Adapted from [109] 
Table 2 Clinical considerations for the management of CVD risk using SGLT2i therapies in people with T2DM $[1,42-48,57-59,77,78,80,81,86]$

\begin{tabular}{|c|c|c|c|c|c|}
\hline $\begin{array}{l}\text { Clinical } \\
\text { consideration }\end{array}$ & $\begin{array}{l}\text { People with T2DM } \\
\text { and established CVD }\end{array}$ & $\begin{array}{l}\text { People with T2DM } \\
\text { and high risk for CVD }\end{array}$ & $\begin{array}{l}\text { People with T2DM } \\
\text { and low risk for CVD }\end{array}$ & $\begin{array}{l}\text { Frail people with } \\
\text { T2DM }\end{array}$ & Evidence level 109 \\
\hline MACE outcomes & & & $\begin{array}{l}\text { May be considered as } \\
\text { preventative }\end{array}$ & & $A+B+E$ \\
\hline $\begin{array}{l}\text { HF hospitalisation } \\
\text { or death }\end{array}$ & & & $\begin{array}{l}\text { May be considered as } \\
\text { primary prevention }\end{array}$ & & $A+B+E$ \\
\hline Blood pressure & & & & & $A+B+E$ \\
\hline Body weight & & & & & $A+B+E$ \\
\hline $\begin{array}{l}\text { Initiation as second } \\
\text { line therapy after } \\
\text { metformin }\end{array}$ & & & & & $\mathrm{E}$ \\
\hline $\begin{array}{l}\text { Switch from second } \\
\text { line therapy that } \\
\text { does not have clear } \\
\text { CVD benefit }\end{array}$ & $\begin{array}{l}\text { Benefit in switching } \\
\text { from agents with } \\
\text { similar glycaemic } \\
\text { control but no proven } \\
\text { CVD benefit } \\
\end{array}$ & $\begin{array}{l}\text { Benefit in switching } \\
\text { from agents with } \\
\text { similar glycaemic } \\
\text { control but no proven } \\
\text { CVD benefit }\end{array}$ & $\begin{array}{l}\text { Benefit in switching } \\
\text { from agents with } \\
\text { similar glycaemic } \\
\text { control but no proven } \\
\text { CVD benefit }\end{array}$ & & $\mathrm{E}$ \\
\hline $\begin{array}{l}\text { Appropriate first } \\
\text { line therapy in } \\
\text { people intolerant to } \\
\text { metformin }\end{array}$ & & & & & $\mathrm{E}$ \\
\hline Thiazide diuretics & & & & & $C+E$ \\
\hline Loop diuretics & & & & & $C+E$ \\
\hline $\begin{array}{l}\text { Diabetic foot or } \\
\text { ulcers }\end{array}$ & & & & & $C+E$ \\
\hline Prior DKA & & & & & E \\
\hline $\begin{array}{l}\text { Initiation at eGFR }<60 \\
\mathrm{~mL} / \mathrm{min}^{\prime} 1.73 \mathrm{m2}^{*}\end{array}$ & & & & & $\begin{array}{l}\text { Outside current } \\
\text { licence }\end{array}$ \\
\hline
\end{tabular}

$C V D$ cardiovascular disease, $D K A$ diabetic ketoacidosis, $e G F R$ estimated glomerular filtration rate, $H F$ heart failure, $M A C E$ major adverse cardiovascular events, T2DM type 2 diabetes mellitus

*SGLT2i therapies may be initiated in people with eGFR $60 \mathrm{~mL} / \mathrm{min} / 1.73 \mathrm{~m}^{2}$. Individuals already treated with canagliflozin or empagliflozin who demonstrate renal decline may continue treatment until eGFR reaches $<45 \mathrm{~mL} / \mathrm{min} / 1.73 \mathrm{~m}^{2}$

appropriate, to treat these people. In people with T2DM and inadequate glucose control, the Committee recommends that an SGLT2i is considered as an early and efficacious treatment option. There is a paucity of RCT data describing the effects of SGLT2 inhibition on CVD outcomes in patients with well-controlled glucose levels, because they were not typically enrolled in the pivotal trials. However, in people with T2DM and established CVD, HCPs may wish to consider the potential CV benefits of SGT2i treatment when reviewing a patient's management and discuss options for maintaining glycaemic control while taking into account comorbid CVD progression.

In people with T2DM and a high risk for CVD, benefits in terms of MACE were demonstrated in CANVAS but not in DECLARE-TIMI 58. However, there were significant reductions in HHF and composites of HF and CV death in both trials; thus, outcomes for people in this stratum are optimised with SGLT2i treatment. Unless contraindicated, the Committee advises that SGLT2is are considered, in combination with lifestyle and other glucose-lowering drugs as appropriate, as adjunctive treatment to improve glucose control and reduce risk of $\mathrm{HF}$ and CVD.

In people with T2DM and a lower risk for CVD, there is less robust evidence available from the trials and studies presented in this paper. However, it is well accepted that T2DM is an independent risk factor for the development of CVD. In addition, lifestyle factors that contribute to the development of T2DM are risk factors for the development of CVD. The Committee advises that for people for whom the HCP can see benefit-in terms of glycaemic control, weight loss, lower blood pressure-that SGLT2i treatment may play a preventative role 
for future HF, while providing efficacious T2DM treatment in the immediate setting. Recent RWE appears to demonstrate HF benefits in patients without established CVD. SGLT2i therapy may also be considered for people at risk of developing CKD, with data supporting efficacy in preventing the worsening of loss of renal function.

In frail people with T2DM, the role of SGLT2i treatment needs to be considered with care. For many of these people, renal impairment will be the main area of concern for both initiation and appropriate discontinuation. For frail people, any secondary effects of weight loss and lowering of blood pressure may be undesirable and must be considered in the personalisation of treatment. The implications of the diuretic action of SGLT2i treatment should be considered when assessing the appropriate treatment and comfort of frail people, especially those with impaired continence. The Committee notes that for frail individuals that are suited to initiation of an SGLT2i treatment, the benefits in HF and MACE outcomes, in an anti-diabetes treatment option that does not increase the risk of hypoglycaemia, may be of value in the optimisation of treatment.

The Improving Diabetes Steering Committee awaits forthcoming evidence for SGLT2i therapy, particularly in terms of further elucidation of CVD benefits in different patient populations (such as the small group of people with T2DM but without CV risk factors [hypertension, dyslipidaemia or smoking] other than diabetes) and in terms of renal benefits. The Committee looks forward to providing further evidence-based advice to primary care HCPs in future updates.

\section{ACKNOWLEDGEMENTS}

Funding. The Improving Diabetes Steering Committee is supported by an educational grant from Napp Pharmaceuticals Limited. Napp has reviewed this document for factual accuracy only. All authors had full access to the articles reviewed in this manuscript and take full responsibility for the integrity and accuracy of this manuscript. The Rapid Service Fee for this study was funded by Napp Pharmaceuticals Limited.

Medical Writing and Additional Assistance. Medical writing services were provided on behalf of the Steering Committee by Dr. Colin Griffin (Griffin Scientific Limited) and Cello Health Communications. Support for this assistance was funded by Napp Pharmaceuticals Limited. The paper has also been reviewed by Professor Miles Fisher, Department of Diabetes, Glasgow Royal Infirmary, UK.

Authorship. All named authors meet the International Committee of Medical Journal Editors (ICMJE) criteria for authorship of this manuscript, take responsibility for the integrity of the work as a whole, and have given final approval for the version to be published.

Disclosures. Dr. Amar Ali has received speaker honoraria from all current manufacturers of SGLT2 inhibitors. Professor Steve Bain has received research support from Healthcare and Research Wales (Welsh Government), and honoraria from Boehringer Ingelheim, Lilly, Merck, Napp Pharmaceuticals Ltd, Novo Nordisk and Sanofi. He has ownership interest in Glycosmedia (diabetes on-line news service). He is also a member of this journal's Editorial Board. Dr. Marc Evans has received research support from Novo Nordisk and Takeda. He has received speaker fees and honoraria from Boehringer Ingelheim, Janssen, MSD, Napp Pharmaceuticals Ltd, Novartis and Novo Nordisk. He is also the Editor-in-Chief of this journal. Dr. Kevin Fernando has received speaker honoraria from all current manufacturers of SGLT2 inhibitors. Debbie Hicks has received speaker honoraria from Roche, Sanofi and Takeda. She has received speaker and advisory board honoraria from Abbott, AstraZeneca, BD, Boehringer Ingelheim, Janssen, Lilly, MSD, Mylan, Napp Pharmaceuticals Ltd and Novo Nordisk. Educational sponsorship has been provided by $\mathrm{BD}$, Boehringer Ingelheim, Lilly, MSD, Mylan and Novo Nordisk. She has also received conference registration and subsistence from BD, Lilly and Novo Nordisk. Debbie does 
not hold any shares in any pharmaceutical company. June James has received funding for provision of educational sessions, resources and attendance at advisory boards from AstraZeneca, BD, Boehringer Ingelheim, Janssen, Lilly, MSD, Napp Pharmaceuticals Ltd, Novo Nordisk and Sanofi. Nicola Milne has received funding for provision of educational sessions or attendance at advisory boards from AstraZeneca, BD, Boehringer Ingelheim, Janssen, Lilly, MSD, Napp Pharmaceuticals Ltd, Novo Nordisk, Roche, Sanofi and Takeda. She has received travel grants to attend conferences from AstraZeneca, Boehringer Ingelheim, Novo Nordisk, Napp Pharmaceuticals Ltd and Novartis. Philip Newland-Jones has conducted research studies funded by, served as advisor to, and received lecture honoraria and travel support from, AstraZeneca, Boehringer Ingelheim, Lilly, Janssen, Lifescan, MSD, Mylan, Napp Pharmaceuticals Ltd, Novo Nordisk, Sanofi, Servier and Takeda. Dr. Dipesh Patel has received educational sponsorship, speaker or advisory related honoraria from AstraZeneca, Boehringer Ingelheim, Janssen, Lilly, Napp Pharmaceuticals Ltd, Novo Nordisk, Sanofi, MSD and Takeda. Dr. Adie Viljoen has conducted research studies funded by, served as advisor to, and received lecture honoraria and travel support from, Amgen, AstraZeneca, Boehringer Ingelheim, Lilly, MSD, Napp Pharmaceuticals Ltd, Novo Nordisk, Pfizer, Regeneron, Sanofi Aventis and Tosoh. Professor John Wilding has received funding for providing educational sessions and/ or attending advisory boards from Astellas, AstraZeneca, Biologix, Boehringer Ingelheim, Janssen, Lilly, Merck, Mundipharma, Napp Pharmaceuticals Ltd, Novo Nordisk, Orexigen, Sanofi and Takeda. He has received travel grants to attend conferences from AstraZeneca, Boehringer Ingelheim, Lilly, Napp Pharmaceuticals Ltd, Novo Nordisk and Sanofi. He has received institutional research grant support or funding for clinical trials from AstraZeneca, Boehringer Ingelheim, Janssen, Novo Nordisk, Sanofi and Takeda.

Compliance with Ethics Guidelines. The article is based on previously conducted studies and does not contain any new studies with human participants or animals.

Open Access. This article is distributed under the terms of the Creative Commons Attribution-NonCommercial 4.0 International License (http://creativecommons.org/licenses/ by-nc/4.0/), which permits any noncommercial use, distribution, and reproduction in any medium, provided you give appropriate credit to the original author(s) and the source, provide a link to the Creative Commons license, and indicate if changes were made.

\section{REFERENCES}

1. Wilding J, Fernando K, Milne N, et al. SGLT2 inhibitors in type 2 diabetes management: key evidence and implications for clinical practice. Diabetes Ther. 2018;9:1757-73.

2. Pantalone KM, Hobbs TM, Wells BJ, et al. Clinical characteristics, complications, comorbidities and treatment patterns among patients with type 2 diabetes mellitus in a large integrated health system. BMJ Open Diabetes Res Care. 2015;3:e000093.

3. Einarson TR, Acs A, Ludwig C, Panton UH. Prevalence of cardiovascular disease in type 2 diabetes: a systematic literature review of scientific evidence from across the world in 2007-2017. Cardiovasc Diabetol. 2018;17:83.

4. Shah AD, Langenberg C, Rapsomaniki E, et al. Type 2 diabetes and incidence of cardiovascular diseases: a cohort study in 1.9 million people. Lancet Diabetes Endocrinol. 2015;3:105-13.

5. World Health Organization. Fact sheets: cardiovascular diseases (CVDs). 2017. Retrieved from: https:// www.who.int/en/news-room/fact-sheets/detail/car diovascular-diseases-(cvds).

6. Einarson TR, Acs A, Ludwig C, Panton UH. Economic burden of cardiovascular disease in type 2 diabetes: a systematic review. Value Health. 2018;21:881-90.

7. De Rosa S, Arcidiacono B, Chiefari E, Brunetti A, Indolfi C, Foti DP. Type 2 diabetes mellitus and cardiovascular disease: genetic and epigenetic links. Front Endocrinol (Lausanne). 2018;9:2.

8. Stern MP. Diabetes and cardiovascular disease. The "common soil" hypothesis. Diabetes. 1995;44:369-74. 
9. Leon BM, Maddox TM. Diabetes and cardiovascular disease: epidemiology, biological mechanisms, treatment recommendations and future research. World J Diabetes. 2015;6:1246-58.

10. Gonzalez N, Moreno-Villegas Z, Gonzalez-Bris A, Egido J, Lorenzo O. Regulation of visceral and epicardial adipose tissue for preventing cardiovascular injuries associated to obesity and diabetes. Cardiovasc Diabetol. 2017;16:44.

11. Ortega FB, Lavie CJ, Blair SN. Obesity and cardiovascular disease. Circ Res. 2016;118:1752-70.

12. Lalia AZ, Dasari S, Johnson ML, et al. Predictors of whole-body insulin sensitivity across ages and adiposity in adult humans. J Clin Endocrinol Metab. 2016;101:626-34.

13. Laakso M, Kuusisto J. Insulin resistance and hyperglycaemia in cardiovascular disease development. Nat Rev Endocrinol. 2014;10:293-302.

14. Ormazabal V, Nair S, Elfeky O, Aguayo C, Salomon $\mathrm{C}$, Zuniga FA. Association between insulin resistance and the development of cardiovascular disease. Cardiovasc Diabetol. 2018;17:122.

15. Brannick B, Dagogo-Jack S. Prediabetes and cardiovascular disease: pathophysiology and interventions for prevention and risk reduction. Endocrinol Metab Clin North Am. 2018;47:33-50.

16. DeFronzo RA, Abdul-Ghani M. Assessment and treatment of cardiovascular risk in prediabetes: impaired glucose tolerance and impaired fasting glucose. Am J Cardiol. 2011;108:3B-24B.

17. Ryden L, Standl E, Bartnik M, et al. Guidelines on diabetes, pre-diabetes, and cardiovascular diseases: executive summary. The Task Force on Diabetes and Cardiovascular Diseases of the European Society of Cardiology (ESC) and of the European Association for the Study of Diabetes (EASD). Eur Heart J. 2007;28:88-136.

18. Ginsberg HN. Insulin resistance and cardiovascular disease. J Clin Invest. 2000;106:453-8.

19. Lee JS, Chang PY, Zhang Y, Kizer JR, Best LG, Howard BV. Triglyceride and HDL-C dyslipidemia and risks of coronary heart disease and ischemic stroke by glycemic dysregulation status: the strong heart study. Diabetes Care. 2017;40:529-37.

20. Schofield JD, Liu Y, Rao-Balakrishna P, Malik RA, Soran H. Diabetes dyslipidemia. Diabetes Ther. 2016;7:203-19.

21. Rungby J, Schou M, Warrer P, Ytte L, Andersen GS. Prevalence of cardiovascular disease and evaluation of standard of care in type 2 diabetes: a nationwide study in primary care. Cardiovasc Endocrinol. 2017;6:145-51.

22. Packer M. Heart failure: the most important, preventable, and treatable cardiovascular complication of type 2 diabetes. Diabetes Care. 2018;41:11-3.

23. Cefalu WT, Kaul S, Gerstein HC, et al. Cardiovascular outcomes trials in type 2 diabetes: where do we go from here? Reflections from a diabetes care editors' expert forum. Diabetes Care. 2018;41:14-31.

24. Velagaleti RS, Vasan RS. Heart failure in the twentyfirst century: is it a coronary artery disease or hypertension problem? Cardiol Clin. 2007;25:487-95.

25. Rosano GM, Vitale C, Seferovic P. Heart failure in patients with diabetes mellitus. Card Fail Rev. 2017;3:52-5.

26. McMurray JJ, Gerstein HC, Holman RR, Pfeffer MA. Heart failure: a cardiovascular outcome in diabetes that can no longer be ignored. Lancet Diabetes Endocrinol. 2014;2:843-51.

27. Seferovic PM, Petrie MC, Filippatos GS, et al. Type 2 diabetes mellitus and heart failure: a position statement from the Heart Failure Association of the European Society of Cardiology. Eur J Heart Fail. 2018;20:853-72.

28. Hancock HC, Close H, Mason JM, et al. High prevalence of undetected heart failure in long-term care residents: findings from the Heart Failure in Care Homes (HFinCH) study. Eur J Heart Fail. 2013;15:158-65.

29. Di Lenarda A, Stellato K, Radini D. The challenge of heart failure diagnosis and management in primary care in elderly population: mere illusion or concrete opportunity? Int J Cardiol. 2019;274:248-9.

30. Van Linthout S, Tschope C. Inflammation-cause or consequence of heart failure or both? Curr Heart Fail Rep. 2017;14:251-65.

31. Thomas MC. Type 2 diabetes and heart failure: challenges and solutions. Curr Cardiol Rev. 2016;12:249-55.

32. Kasznicki J, Drzewoski J. Heart failure in the diabetic population-pathophysiology, diagnosis and management. Arch Med Sci. 2014;10:546-56.

33. Matsushita K, Blecker S, Pazin-Filho A, et al. The association of hemoglobin A1c with incident heart failure among people without diabetes: the atherosclerosis risk in communities study. Diabetes. 2010;59:2020-6. 
34. Carr AA, Kowey PR, Devereux RB, et al. Hospitalizations for new heart failure among subjects with diabetes mellitus in the RENAAL and LIFE studies. Am J Cardiol. 2005;96:1530-6.

35. Tenenbaum A, Motro M, Fisman EZ, et al. Functional class in patients with heart failure is associated with the development of diabetes. Am J Med. 2003;114:271-5.

36. Conrad N, Judge A, Tran J, et al. Temporal trends and patterns in heart failure incidence: a population-based study of 4 million individuals. Lancet. 2018;391:572-80.

37. Selvin E, Marinopoulos S, Berkenblit G, et al. Metaanalysis: glycosylated hemoglobin and cardiovascular disease in diabetes mellitus. Ann Intern Med. 2004;141:421-31.

38. Stratton IM, Adler AI, Neil HA, et al. Association of glycaemia with macrovascular and microvascular complications of type 2 diabetes (UKPDS 35): prospective observational study. BMJ. 2000;321:405-12.

39. Yudkin JS, Richter B, Gale EA. Intensified glucose lowering in type 2 diabetes: time for a reappraisal. Diabetologia. 2010;53:2079-85.

40. Selvin E, Coresh J, Golden SH, Brancati FL, Folsom AR, Steffes MW. Glycemic control and coronary heart disease risk in persons with and without diabetes: the atherosclerosis risk in communities study. Arch Intern Med. 2005;165:1910-6.

41. Zhao W, Katzmarzyk PT, Horswell R, Wang Y, Johnson J, Hu G. HbA1c and coronary heart disease risk among diabetic patients. Diabetes Care. 2014;37:428-35.

42. Ruospo M, Saglimbene VM, Palmer SC, et al. Glucose targets for preventing diabetic kidney disease and its progression. Cochrane Database Syst Rev. 2017;6:CD010137.

43. Turnbull FM, Abraira C, Anderson RJ, et al. Intensive glucose control and macrovascular outcomes in type 2 diabetes. Diabetologia. 2009;52:2288-98.

44. Turner RC, Millns H, Neil HA, et al. Risk factors for coronary artery disease in non-insulin dependent diabetes mellitus: United Kingdom Prospective Diabetes Study (UKPDS: 23). BMJ. 1998;316:823-8.

45. Action to Control Cardiovascular Risk in Diabetes Study Group, Gerstein HC, Miller ME, et al. Effects of intensive glucose lowering in type 2 diabetes. N Engl J Med. 2008;358:2545-59.

46. Group AC, Patel A, MacMahon S, et al. Intensive blood glucose control and vascular outcomes in patients with type 2 diabetes. $\mathrm{N}$ Engl J Med. 2008;358:2560-72.

47. Duckworth W, Abraira C, Moritz T, et al. Glucose control and vascular complications in veterans with type 2 diabetes. N Engl J Med. 2009;360:129-39.

48. Andersson C, Olesen JB, Hansen PR, et al. Metformin treatment is associated with a low risk of mortality in diabetic patients with heart failure: a retrospective nationwide cohort study. Diabetologia. 2010;53:2546-53.

49. Mannucci E, Dicembrini I, Lauria A, Pozzilli P. Is glucose control important for prevention of cardiovascular disease in diabetes? Diabetes Care. 2013;36(Suppl 2):S259-63.

50. Zinman B, Wanner C, Lachin JM, et al. Empagliflozin, cardiovascular outcomes, and mortality in type 2 diabetes. N Engl J Med. 2015;373:2117-28.

51. Neal B, Perkovic V, Mahaffey KW, et al. Canagliflozin and cardiovascular and renal events in type 2 diabetes. N Engl J Med. 2017;377:644-57.

52. Wiviott SD, Raz I, Bonaca MP, et al. Dapagliflozin and cardiovascular outcomes in type 2 diabetes. N Engl J Med. 2019;380:347-57.

53. Cavender MA, Norhammar A, Birkeland KI, et al. SGLT-2 inhibitors and cardiovascular risk: an analysis of CVD-REAL. J Am Coll Cardiol. 2018;71:2497-506.

54. Kosiborod M, Cavender MA, Fu AZ, et al. Lower risk of heart failure and death in patients initiated on sodium-glucose cotransporter-2 inhibitors versus other glucose-lowering drugs: the CVD-REAL study (Comparative Effectiveness of Cardiovascular Outcomes in New Users of Sodium-Glucose Cotransporter-2 Inhibitors). Circulation. 2017;136:249-59.

55. Ryan PB, Buse JB, Schuemie MJ, et al. Comparative effectiveness of canagliflozin, SGLT2 inhibitors and non-SGLT2 inhibitors on the risk of hospitalization for heart failure and amputation in patients with type 2 diabetes mellitus: a real-world meta-analysis of 4 observational databases (OBSERVE-4D). Diabetes Obes Metab. 2018;20:2585-97.

56. Udell JA, Yuan Z, Rush T, Sicignano NM, Galitz M, Rosenthal N. Cardiovascular outcomes and risks after initiation of a sodium glucose cotransporter 2 inhibitor: results from the EASEL population-based cohort study (Evidence for Cardiovascular Outcomes With Sodium Glucose Cotransporter 2 Inhibitors in the Real World). Circulation. 2018;137:1450-9.

57. Inzucchi SE, Zinman B, Wanner C, et al. SGLT-2 inhibitors and cardiovascular risk: proposed 
pathways and review of ongoing outcome trials. Diab Vasc Dis Res. 2015;12:90-100.

58. Heerspink HJ, Perkins BA, Fitchett DH, Husain M, Cherney DZ. Sodium glucose cotransporter 2 inhibitors in the treatment of diabetes mellitus: cardiovascular and kidney effects, potential mechanisms, and clinical applications. Circulation. 2016;134:752-72.

59. Verma S, McMurray JJV. SGLT2 inhibitors and mechanisms of cardiovascular benefit: a state-ofthe-art review. Diabetologia. 2018;61:2108-17.

60. Ferrannini E, Mark M, Mayoux E. CV protection in the EMPA-REG OUTCOME trial: a "thrifty substrate" hypothesis. Diabetes Care. 2016;39:1108-14.

61. Rastogi A, Bhansali A. SGLT2 inhibitors through the windows of EMPA-REG and CANVAS trials: a review. Diabetes Ther. 2017;8:1245-51.

62. Kelly MS, Lewis J, Huntsberry AM, Dea L, Portillo I. Efficacy and renal outcomes of SGLT2 inhibitors in patients with type 2 diabetes and chronic kidney disease. Postgrad Med. 2019;131:31-42.

63. Seidu S, Kunutsor SK, Cos X, et al. SGLT2 inhibitors and renal outcomes in type 2 diabetes with or without renal impairment: a systematic review and meta-analysis. Prim Care Diabetes. 2018;12:265-83.

64. Wanner C, Lachin JM, Inzucchi SE, et al. Empagliflozin and clinical outcomes in patients with type 2 diabetes mellitus, established cardiovascular disease, and chronic kidney disease. Circulation. 2018;137:119-29.

65. Neal B, Perkovic V, Mahaffey KW, et al. Optimizing the analysis strategy for the CANVAS Program: a prespecified plan for the integrated analyses of the CANVAS and CANVAS-R trials. Diabetes Obes Metab. 2017;19:926-35.

66. AstraZeneca UK Limited. Dapagliflozin summary of product characteristics. 2018. https://www. medicines.org.uk/emc/product/7607. Accessed Apr 2018.

67. Boehringer Ingelheim Limited. Empagliflozin summary of product characteristics. 2018. https://www. medicines.org.uk/emc/product/5441. Accessed May 2019.

68. Napp Pharmaceuticals Limited. Canagliflozin summary of product characteristics. 2018. https://www. medicines.org.uk/emc/product/8855. Accessed May 2019.

69. Jardine MJ, Mahaffey KW, Neal B, et al. The Canagliflozin and Rrenal Endpoints in Diabetes with Established Nephropathy Clinical Evaluation
(CREDENCE) study rationale, design, and baseline characteristics. Am J Nephrol. 2017;46:462-72.

70. Johnson and Johnson. Phase 3 CREDENCE Renal Outcomes Trial of INVOKANA ${ }^{\circledR}$ is Being Stopped Early for Positive Efficacy Findings. 2018. Retrieved from: https://www.jnj.com/phase-3-credence-renaloutcomes-trial-of-invokana-canagliflozin-is-beingstopped-early-for-positive-efficacy-findings.

71. U.S. Food and Drug Administration. Guidance document: Diabetes Mellitus - Evaluating cardiovascular risk in new antidiabetic therapies to treat type 2 diabetes. 2008. Retrieved from: https://www. fda.gov/regulatory-information/search-fda-guidanc e-documents/diabetes-mellitus-evaluating-cardiova scular-risk-new-antidiabetic-therapies-treat-type-2diabetes.

72. Nissen SE, Wolski K. Effect of rosiglitazone on the risk of myocardial infarction and death from cardiovascular causes. N Engl J Med. 2007;356: 2457-71.

73. Schweizer A, Dejager S, Foley JE, Couturier A, Ligueros-Saylan M, Kothny W. Assessing the cardiocerebrovascular safety of vildagliptin: meta-analysis of adjudicated events from a large phase III type 2 diabetes population. Diabetes Obes Metab. 2010;12:485-94.

74. White WB, Pratley R, Fleck P, et al. Cardiovascular safety of the dipetidyl peptidase- 4 inhibitor alogliptin in type 2 diabetes mellitus. Diabetes Obes Metab. 2013;15:668-73.

75. Scirica BM, Bhatt DL, Braunwald E, et al. Saxagliptin and cardiovascular outcomes in patients with type 2 diabetes mellitus. N Engl J Med. 2013;369:1317-26.

76. Engel SS, Golm GT, Shapiro D, Davies MJ, Kaufman $\mathrm{KD}$, Goldstein BJ. Cardiovascular safety of sitagliptin in patients with type 2 diabetes mellitus: a pooled analysis. Cardiovasc Diabetol. 2013;12:3.

77. Clifton P. Do dipeptidyl peptidase IV (DPP-IV) inhibitors cause heart failure? Clin Ther. 2014;36:2072-9.

78. Monami M, Dicembrini I, Mannucci E. Dipeptidyl peptidase-4 inhibitors and heart failure: a metaanalysis of randomized clinical trials. Nutr Metab Cardiovasc Dis. 2014;24:689-97.

79. Marso SP, Daniels GH, Brown-Frandsen K, et al. Liraglutide and cardiovascular outcomes in type 2 diabetes. N Engl J Med. 2016;375:311-22.

80. Marso SP, Bain SC, Consoli A, et al. Semaglutide and cardiovascular outcomes in patients with type 2 diabetes. N Engl J Med. 2016;375:1834-44. 
81. Hernandez AF, Green JB, Janmohamed S, et al. Albiglutide and cardiovascular outcomes in patients with type 2 diabetes and cardiovascular disease (Harmony Outcomes): a double-blind, randomised placebo-controlled trial. Lancet. 2018;392:1519-29.

82. Lilly. Trulicity ${ }^{\circledR}$ (dulaglutide) demonstrates superiority in reduction of cardiovascular events for broad range of people with type 2 diabetes. 2018. https:// investor.lilly.com/news-releases/news-releasedetails/trulicityr-dulaglutide-demonstratessuperiority-reduction 2018 .

83. Holman RR, Bethel MA, Mentz RJ, et al. Effects of once-weekly exenatide on cardiovascular outcomes in type 2 diabetes. N Engl J Med. 2017;377:1228-39.

84. Pfeffer MA, Claggett B, Diaz R, et al. Lixisenatide in patients with type 2 diabetes and acute coronary syndrome. N Engl J Med. 2015;373:2247-57.

85. Fitchett D, Butler J, van de Borne P, et al. Effects of empagliflozin on risk for cardiovascular death and heart failure hospitalization across the spectrum of heart failure risk in the EMPA-REG OUTCOME ${ }^{\circledR}$ trial. Eur Heart J. 2018;39:363-70.

86. Fitchett DH. Empagliflozin and cardio-renal outcomes in patients with type 2 diabetes and cardiovascular disease-implications for clinical practice. Eur Endocrinol. 2018;14:40-9.

87. Radholm K, Figtree G, Perkovic V, et al. Canagliflozin and heart failure in type 2 diabetes mellitus. Circulation. 2018;138:458-68.

88. Raz I, Mosenzon O, Bonaca MP, et al. DECLARETIMI 58: participants' baseline characteristics. Diabetes Obes Metab. 2018;20:1102-10.

89. Furtado RHM, Bonaca MP, Raz I, et al. Dapagliflozin and cardiovascular outcomes in patients with type 2 diabetes and previous myocardial infarction: subanalysis from DECLARE TIMI-58 Trial. Circulation. 2019;139(22):2516-27.

90. Kennedy-Martin T, Curtis S, Faries D, Robinson S, Johnston J. A literature review on the representativeness of randomized controlled trial samples and implications for the external validity of trial results. Trials. 2015;16:495.

91. Roche N, Reddel H, Martin R, et al. Quality standards for real-world research. Focus on observational database studies of comparative effectiveness. Ann Am Thorac Soc. 2014;11(Suppl 2):S99-104.

92. Suvarna VR. Real world evidence (RWE)-are we (RWE) ready? Perspect Clin Res. 2018;9:61-3.

93. Camm AJ, Fox KAA. Strengths and weaknesses of 'real-world' studies involving non-vitamin K antagonist oral anticoagulants. Open Heart. 2018;5:e000788.

94. Persson F, Nystrom T, Jorgensen ME, et al. Dapagliflozin is associated with lower risk of cardiovascular events and all-cause mortality in people with type 2 diabetes (CVD-REAL Nordic) when compared with dipeptidyl peptidase-4 inhibitor therapy: a multinational observational study. Diabetes Obes Metab. 2018;20:344-51.

95. McMurray JJV. Only trials tell the truth about treatment effects. J Am Coll Cardiol. 2018;71:2640-2.

96. Boehringer Ingelheim. Initial results from EMPRISE real-world evidence study shows empagliflozin was associated with reduced risk for hospitalisation for heart failure compared with DPP-4 inhibitors in people with type 2 diabetes with and without cardiovascular disease. 2018. Retrieved from: https:// www.boehringer-ingelheim.com/press-release/initi al-results-emprise-real-world-evidence-study.

97. Zelniker TA, Wiviott SD, Raz I, et al. SGLT2 inhibitors for primary and secondary prevention of cardiovascular and renal outcomes in type 2 diabetes: a systematic review and meta-analysis of cardiovascular outcome trials. Lancet. 2019;393:31-9.

98. National Institute for Health and Clinical Excellence (NICE). Type 2 diabetes in adults: management (NG28). 2017. Retrieved from: https://www. nice.org.uk/guidance/ng28.

99. Barnett AH, O’Hare P, Halcox J. Guidelines for type 2 diabetes: keeping a finger on the pulse. Lancet Diabetes Endocrinol. 2017;5:420.

100. Scottish Intercollegiate Guideline Network (SIGN). Pharmacological management of glycaemic control in people with type 2 diabetes. (SIGN 154.). 2017.

101. Davies MJ, D'Alessio DA, Fradkin J, et al. Management of hyperglycemia in type 2 diabetes. A consensus report by the American Diabetes Association (ADA) and the European Association for the Study of Diabetes (EASD). Diabetes Care. 2018;41(12):2669-2701.

102. Khunti K, Wolden ML, Thorsted BL, Andersen M, Davies MJ. Clinical inertia in people with type 2 diabetes: a retrospective cohort study of more than 80,000 people. Diabetes Care. 2013;36:3411-7.

103. Sanon VP, Patel S, Sanon S, Rodriguez R, Pham SV, Chilton R. Differential cardiovascular profiles of sodium-glucose cotransporter 2 inhibitors: critical evaluation of empagliflozin. Ther Clin Risk Manag. 2017;13:603-11. 
104. Opingari E, Partridge ACR, Verma S, Bajaj HS. SGLT2 inhibitors: practical considerations and recommendations for cardiologists. Curr Opin Cardiol. 2018;33:676-82.

105. Kimura G. Diuretic action of sodium-glucose cotransporter 2 inhibitors and its importance in the management of heart failure. Circ J. 2016;80:2277-81.

106. Wilcox CS, Shen W, Boulton DW, Leslie BR, Griffen SC. Interaction between the sodium-glucose-linked transporter 2 inhibitor dapagliflozin and the loop diuretic bumetanide in normal human subjects. J Am Heart Assoc. 2018;7:e007046.
107. Mordi NA, Mordi IR, Singh JS, et al. Renal and Cardiovascular Effects of sodium-glucose cotransporter 2 (SGLT2) inhibition in combination with loop Diuretics in diabetic patients with Chronic Heart Failure (RECEDE-CHF): protocol for a randomised controlled double-blind cross-over trial. BMJ Open. 2017;7:e018097.

108. Fioretto P, Stefansson BV, Johnsson E, Cain VA, Sjostrom CD. Dapagliflozin reduces albuminuria over 2 years in patients with type 2 diabetes mellitus and renal impairment. Diabetologia. 2016;59:2036-9.

109. Introduction: Standards of medical care in diabetes. Diabetes Care. 2018;41:S1-2. 